Magnet i c conf i nement exper i ment s:

pl asma mat er i al i nt er act i ons, di vertors,

I i miters, scrape- of $f$ I ayer (EX/D), st abi I ity

(EX/S), wave- pl astra i nt er act i ons, cur rent

dri ve, heat ing, energet i c parti cl es (EX/W)

\begin{tabular}{|l|l|}
\hline $\begin{array}{l}\text { j our nal or } \\
\text { publ i cat i on } \mathrm{titl} \text { e }\end{array}$ & Nucl ear Fusi on \\
\hline vol une & 53 \\
\hline number & 10 \\
\hline page $\mathrm{r}$ ange & 104025 \\
\hline year & $2013-09-26$ \\
\hline URL & ht t p: //hdl . handl e. net /10655/00012693 \\
\hline
\end{tabular}




\title{
Magnetic confinement experiments: plasma-material interactions, divertors, limiters, scrape-off layer (EX/D), stability (EX/S), wave-plasma interactions, current drive, heating, energetic particles (EX/W)
}

\author{
H.Yamada
}

National Institute for Fusion Science, Toki 509-5292, Japan

\begin{abstract}
This article summarizes the results presented at the 24th IAEA Fusion Energy Conference in the categories of plasma-material interactions, divertors, limiters, scrape-off layer (EX/D), stability (EX/S), wave-plasma interactions, current drive, heating, energetic particles $(\mathrm{EX} / \mathrm{W})$ in magnetic confinement experiments. In total, 149 papers including post-deadline papers have contributed to these categories. Several closely related papers, which are actually categorized in confinement $(\mathrm{EX} / \mathrm{C})$, have also been included. The understanding of experimental results has progressed remarkably, in particular, in the topics of Resonant Magnetic Perturbation and ITER-Like Wall, which are the highlight of this conference. At the same time, identification of the bridging mechanism between the actuator and the consequence still requires further dedicated efforts so as to provide more accurate and reliable extrapolations to ITER and DEMO.
\end{abstract}

\section{Introduction}

The International Program Committee identified the Terms of Reference to summary speakers as 1) Capture the essence of the progress made, 2) What has the fusion community achieved over the past two years ? 3) Where does fusion R\&D stand right now ? 4) Which critical issues, next steps and/or major challenges definitely need focused attention in the immediate future or medium term in order to ensure avoiding gaps and unnecessary delays/surprises on the way towards fusion demonstration ? Drawing attention to the progress since the last 23rd IAEA-FEC in 2010 has led to the fulfillment of these Terms of Reference. The excellent summary of the same categories in the last FEC2010 was made by Dr. Jean Jaquinot [1]. It describes the particular attention that had been given to issues in the critical path of ITER construction. This situation has been accelerated in these last two years. Urgent issues impacting the critical path of the ITER construction are becoming much clearer and the pressure of the demand for more distinct results, which would enable us to choose one among two or more options, is growing. For two examples, one can refer to the in-vessel ELM (Edge Localized Mode) coils and the strategy of a full tungsten divertor in ITER. The last summary for FEC2010 also posed that the experimental results can be qualitatively understood but the extrapolation to ITER requires a more accurate knowledge of the plasma. This summary for FEC2012 confirms the progress in these last two years. 
The presented contributions are summarized along with the highlighted topics; 1.pedestal stability \& control which is primarily focused on ELM mitigation and suppression by Resonant Magnetic Perturbations (RMP), 2. reduction of heat load, 3. plasma wall interaction where the ITER-like wall experiment on JET is a hot topic. And also 4.plasma facing materials, 5. disruptions, 6. effect of rotation on MHD, 7. ST(Spherical Tokamak) startup, 8. plasma scenario and finally 9 . waves and energetic particles.

\section{Pedestal Stability and Control - ELM Mitigation/Suppression and Physics of 3-D Magnetic Fields -}

Control of ELMs is a very serious and emergent issue in ITER [2]. Since the approaches to ELM-free operational regime, pellet-pace making and super-sonic molecular beams are reviewed in the summary of EX/C, an RMP is highlighted here. When an RMP is applied, the toroidal symmetry of a tokamak breaks down and the physics of a 3-D magnetic field emerges. In the last FEC2010, DIII-D[3], JET[4] MAST[5], and NSTX[6] reported related studies. The last summary for FEC2010 [1] pointed out the need to describe accurately the response of the plasma to an RMP and requested a more accurate knowledge of the plasma screening by the RMP. New results from more machines have been reported in the FEC2012. RMPs by in-vessel coils have become newly available in ASDEX-Upgrade[7] and KSTAR[8]. ELM mitigation by an RMP also has been demonstrated for stellartor/heliotron plasma in LHD [9]. In parallel with the demonstration of ELM mitigation and suppression, the remarkable progress in experimental observation of 3-D magnetic fields in these devices should be emphasized

DIII-D has reported RMP ELM suppression which can be extended to the ITER baseline scenario [10]. The $n=3$ perturbation has demonstrated ELM suppression in a regime which matches the ITER target with the same plasma shape and $I_{p} / a B$, and $\beta_{\mathrm{N}}=1.8$ at $v^{*}=0.12$ as shown in Fig.1. The ELM suppression also has been demonstrated in the plasma with a significant fraction of helium $\left(n_{\mathrm{He}} / n_{e}=0.25\right)$. They have identified a change in topology due to an RMP in the experiment by means of X-point soft X-ray imaging (see Fig.2).

ASDEX-Upgrade has reported ELM mitigation with new saddle coils which generate the $n=1,2,4$ perturbation [7]. Type-I ELMs were replaced by frequent small ELMs, which led to significant reduction of the peak divertor heat load and no penalty in energy confinement. In this demonstration in ASDEX-Upgrade, it should be pointed out that the ELM mitigation was insensitive to the resonance condition. 
ELM mitigation has been established on MAST using an RMP with $n=3,4,6[11]$. The ELM frequency increased by up to a factor of 5 with a similar reduction in the energy released by a single ELM. The observation of the X-point lobe has indicated that the vacuum magnetic field gives an over-estimate of the penetration. And one could get good description of the lobe structures if appropriate ideal screening exists at the edge (see Fig.3).

KSTAR has newly demonstrated ELM suppression by an RMP [8]. ELMs were suppressed at $n=1$ for the first time. In this case, the density was pumped out initially by $10 \%$ and the stored energy dropped by $8 \%$. They have also reported no mitigation with non-resonant anti-phase. In their study, ECE Imaging was highlighted as a powerful tool to identify what happens in edge plasmas, which showed that a filamentary structure disappeared when the ELM was suppressed [12] (see Fig.4).

From JET, the progress of ELM mitigation by perturbation coils located outside of the vacuum vessel [13] has been reported. The type-I ELM changed to small and high-frequency (few hundreds Hz) ELMs in the high collisionality of $v_{\mathrm{e}}{ }^{*}=2.0$ at the pedestal while the increase of the ELM frequency was limited to $80 \mathrm{~Hz}$ in a low collisionality of $v_{\mathrm{e}}{ }^{*}=0.8$, which suggested saturation of the effect. ELM mitigation by using external RMP coils also has been reported from LHD [9], where the interchange modes drove ELMs instead of peeling ballooning modes. In this case, it should be pointed out that the resonance was located in the intrinsically stochastic edge layer which surrounds the nested flux surfaces.

Available experimental observations with an external non-axisymmetric magnetic perturbation, which includes 'R'MP, are summarized in Table I. The inevitability of "resonance" was not convincing and an overview of the results has suggested that the onset of island-formation/magnetic braiding may not be essential for the ELM control by 'R'MP coils. The mechanism of ELM suppression/mitigation by means of non-axisymmetric magnetic perturbation awaits further physical clarification. Quantitative understanding of the plasma response to these non-axisymmetric magnetic perturbations has been progressing, in particular, due to fine diagnostics and comparison with numerical computations, which showed clearly that plasma modifies the vacuum magnetic field. These results suggest an upgrade of the design base (e.g. in ITER) in terms of the Chirikov parameter [14].

With regard to ELMs, it should be noted that the first divertor ion temperature measurement has become available in MAST[15] and ASDEX-Upgrade[16], which improved the evaluation of the heat load in the presence of ELMs.

The importance of the plasma response to 3-D magnetic fields is growing and the 3-D plasma 
response to magnetic structure has been discussed in $\operatorname{LHD}[17,18]$ which is intrinsically 3-D because of its stellartor/heliotron configuration. Comparison between the radial electric field $E_{r}$ gradient and the 3-D MHD equilibrium modeling by the HINT2[19] has indicated that the transition point to a strong $E_{r}$ gradient is a potential good index of the last closed flux surface[17] (see Fig.5). LHD has also reported the experimental observation which suggests that RMP penetration depends on stability criteria such magnetic shear and/or magnetic well/hill[18] (see Fig.6).

Other interesting effects of non-axisymmetric fields on plasma have been reported from JET [20] and EXTRAP T2R [21] and the effect on fast-ion redistribution and loss has been discussed in a multi-machine study of ASDEX-Upgrade, DIII-D and KSTAR [22]. Analysis of the 3-D magnetic field based on detailed diagnostics has been conducted on a high-beta tokamak [23] and RFPs [24,25]

Besides the RMP, NSTX has reported the effect of pre-discharge lithium on ELMs [26]. With an increase of lithium deposition, global parameters generally improve and the ELM frequency declined to zero, and eventually ELMs were stabilized. This was explained by stability improvement due to edge plasma modification by wall conditioning.

\section{Reduction of Heat Load}

With regard to the reduction of the heat load on the in-vessel components, in particular, on divertor targets, remarkable progress has been seen in advanced divertors, novel efforts to reduce heat load, studies towards accurate evaluation of heat load and suppression of impurity contamination. Innovative divertor concepts: Super-X and Snowflake have been investigated in MAST[15], NSTX[27], TCV[28] and DIII-D[29]. The initial experiment of the closed helical divertor has been reported from LHD [30]. Demonstrations of approaches to reduce heat load by means of impurity seeding [31-33] and geometrical modification [34] including application of non-axisymmetric 3-D fields [35,36] have been reported. The progress of quantitative assessment towards an accurate evaluation of the heat load also has been remarkable [15,16,37-40].

The snowflake divertor for mitigation of the divertor heat flux has been reported from NSTX [27] and TCV [28]. Both experiments have succeeded in the redistribution of divetor heat flux and demonstrated significant reduction in divertor heat load. The divertor heat flux width strongly decreased as $I_{p}$ increased in NSTX, DIII-D and C-Mod [41]. The snowflake divertor experiment in NSTX with $P_{N B I}=4 \mathrm{MW}, P_{S O L}=3 \mathrm{MW}$ ) has demonstrated significant reduction in divertor heat flux (from 3-7 to $0.5-1 \mathrm{MW} / \mathrm{m}^{2}$ ) [27]. In TCV, the inverted AXUV radiation 
imaging has clearly shown the power redistribution to secondary strike points as the shape transitioned from a single-null configuration to a snowflake configuration. Eventually, the peak ELM heat load on the primary strike point was reduced by a factor of 3.5 [28]. DIII-D has also demonstrated that the snowflake divertor configuration strongly reduced the peak divertor heat flux and increased radiative volume. It should be noted that ELMs are mitigated in the snowflake divertor configuration in DIII-D [29] (see Fig.7).

The influence of perturbation coils on divertor heat load has been reported from LHD [35] and ASDEX-Upgrade [32]. In LHD, an RMP stabilized the radiative divertor in high density operation while the case without an RMP underwent radiation collapse. In the case with an RMP, radiation increased by a factor of three without degradation of core confinement and divertor heat load was reduced by a factor of $3 \sim 10$. The limited zone around the X-point of the remnant $m=1 / n=1$ island was selectively cooled [35] (see Fig.8). In ASDEX-Upgrade, an RMP reduced the peak power load and makes the strike line split up [32]. In both experiment, validation of the 3-D simulation of edge plasmas has been promoted, which facilitates understanding of the plasma response to an RMP. Toroidally non-axisymmetric 2-D heat flux profile has been investigated for natural ELMs and ELMs triggered by the application of a non-axisymmetric magnetic perturbation in NSTX. The rate of degree of asymmetry seemed to be related to the phase locking of the heat flux profile to the external 3-D field [36].

\section{Plasma Wall Interaction - ITER-Like Wall -}

This topic can be rephrased as the ITER-Like Wall (ILW) in this FEC2012. The in-vessel components in JET have been converted to those like ITER, i.e., beryllium wall and tungsten divertor, while keeping the previous geometrical configuration when carbon wall and divertor was used. This careful modification enabled assessment of the effect of the ILW by comparing the data with the carbon wall and divertor [42].

JET has reported very encouraging result in terms of fuel retention. Retention with the ILW was reduced by one order of magnitude from that with CFC [43] (see Fig.9). The main mechanism was attributed to a change in co-deposition. Be-layers have a lower fuel content than a C-layer. This reduction of retention is in line with the predictions for ITER from CFC to Be/W walls [44] and could secure 1,250 DT discharge (400s) in ITER before cleaning.

The multi machine scaling of the retention rate has been derived through the efforts of the ITPA, which suggested that the retention rate during a shot is expected to range from $\sim 1 \mathrm{~g}$ of $\mathrm{T} /$ hour in full tungsten up to $\sim 100 \mathrm{~g}$ of T/hour in full carbon for a nominal ITER shot [45]. 
Characterization of the initial breakdowns of plasma discharges has been carried out for operations with carbon and the ILW in JET [46]. Non assisted breakdown has been demonstrated to down to an ITER value as low as $0.35 \mathrm{~V} / \mathrm{m}$. Resultant lower radiation levels at higher density have been achieved making the breakdown more robust. Unlike the C-wall, no de-conditioning event following disruptions has been observed with the ILW. Neither glow discharge cleaning nor Be evaporation during operation are longer needed. These preferable consequences were attributed to the drop of carbon by a factor of 20 from CFC to ILW [47] (see Fig.10).

The investigation of plasma heating under an ILW will be discussed in the Sec.9.

\section{Plasma Facing Material -Wall Conditioning and Basic Research of Materials -}

Although plasma facing material issues used to be out of the scope the preceding series of this conference, it has become a critical subject, which involves wall conditioning and basic research of materials.

With regard to wall conditioning, international research on ion cyclotron wall conditioning coordinated by ITPA has been promoted, in particular, for the application of ICRF since glow discharge cleaning is not applicable to steady-state superconducting machines like ITER. The database has been integrated into the ITER baseline using the planned ICRF heating system, and into experimental and modeling efforts coordinated by ITPA [48].

Lithium coating has been investigated in many experiments such as T-11M [49], NSTX [26,50,51], TJ-II [52], RFX [53] and EAST [54] to improve plasma performance and to evaluate the prospects of an application to DEMO. The importance of wall conditioning and the wall material to plasma performance has been suggested for a few decades. For example, the characteristic differences of H-mode with carbon and metal walls have been investigated in ASDEX [55] and a theoretical model to explain these differences has been proposed [56]. The extrapolation to ITER requires a more accurate knowledge of this plasma-wall issue, and the comprehensive assessment of new results from the ILW on JET, these lithium experiments and the other metal wall experiments including the past experiments could lead to the sought resolution.

In this topic, the importance of small basic experiment which used to be likely out of the scope of this FEC should be emphasized in two approaches. The first point is the basic characterization of materials. Since tungsten forms a FUZZ structure with helium, the consequences such as dust production are a concern. Basic research provides confidence that 
key growth parameters, from smaller devices [57-62], can be used for prediction in future devices. For example, tungsten nano-tendril growth has been investigated in Alcator C-mod [57] (see Fig.11) and arcing on carbon and tungsten materials has been compared on JT-60U, LHD and NAGDIS-II [58]. The second point is the development of in-situ measurements of the Plasma Wall Interaction (PWI). Postmortem assessment apparently is not sufficient for understanding PWI processes. For example, qualitative local fuel retention is evaluated by the combination of laser induced fuel desorption by spot laser heating and spectroscopic detection in edge plasmas in TEXTOR [63]. In Alactor C-Mod, a $1 \mathrm{MeV}$ deuteron ion beam has been installed for the time-resolved, in-situ measurement of boron coating and fuel retention [64]. Substantial use of these in-situ methodologies in large magnetic confinement experiments is expected to bridge the basic understanding and characterization in reactor relevant environment.

It would be said that dust [59] and fuel removal [65] remain areas where great effort is still needed for the future success of ITER and DEMO.

\section{Disruptions - Runaway Electrons, Stabilization \& Mitigation and Prediction -}

Disruption is a particularly harmful event in tokamak operation as well as ELMs. Steady and solid progress has been made in the key elements of runaway electrons [66-71], stabilization and mitigation [72-75], and prediction [76-78].

With regard to runaway electrons, for example, DIII-D experiments provided the physics basis for runaway electron control in ITER. Radial stability provided time to dissipate runaways, which enable high- $Z$ gas injection to increase dissipation of runaway electrons [66] (see Fig.12). In ToreSupra, a new concept of Neon gas injection by means of a bursting disk cartridge injector has been demonstrated [67].

In JET, the dynamics of disruptions were very different with the ILW due to higher plasma purity and consequent lower radiation during disruption. Massive gas injection as a disruption mitigation tool is now mandatory for JET. With the mitigation by MGI, the forces and power loads resulting from disruptions are returned to the levels with a $\mathrm{C}$ wall [72] (see Fig.13). It should also be noted that no runaway electron formation has been observed with the ILW [68]

In NSTX, low disruptivity at a relatively high $\beta_{\mathrm{N}}$ as high as 6 with low $1_{i}$ has been found from the vast database. This empirical observation was consistent with specific disruption control experiments. The operational envelope on the plane of Resonant Field Amplification and $\beta_{N} / l_{i}$ 
showed a peak around $\beta_{\mathrm{N}} / l_{\mathrm{i}}$ of 10 [73] (see Fig.14). The disruption warning algorithm showed a high probability of success. $\sim 98 \%$ of disruptions can be flagged with at least a $10 \mathrm{~ms}$ warning [76].

\section{Effect of Flows on MHD Instability}

Several MHD studies have discussed the effect of flows on instabilities such as neo-classical tearing modes (NTM) and sawteeth [79-84]. Plasma rotation alteration and MHD stability have been investigated in KSTAR. The H-mode operation of KSTAR has been expanded toward higher $\beta_{\mathrm{N}}$ and lower $l_{\mathrm{i}}$ and $3 / 2$ and $2 / 1$ tearing modes were characterized with their dependence on plasma rotation (see Fig.15). Alteration of rotation profile has been demonstrated by the application of $n=1,2$ non-axisymmetric fields [79]. In Alcator C-mod, NTMs have been observed in ICRF heated high-performance I-mode plasmas. The NTMs slowed down plasma rotation, which is intrinsic rotation driven by ICRF mode conversion, and also affected plasma performance [80].

\section{ST Start-up}

Efficient start-up of a Spherical Tokamak (ST) is prerequisite for the next generation ST, therefore, non-inductive start-up and current-drive using lower hybrid waves [85,86], electron cyclotron waves [87], electron Bernstein waves [88] and helicity injection [89,90] have been intensively studied. The development of non-inductive current drive by LHCD has been implemented in Globus $M$ and this scenario will be the focal point of the next program Globus-M2 [85]. NSTX has demonstrated that L-mode discharge ramping to 1MA required $35 \%$ less inductive flux when coaxial helicity injection was used [89] (see Fig. 16).

With regard to the heating of ST, ST merging produced bi-directional outflows, causing reconnection heating with high-power up to 5MW. It has been demonstrated that a low- $\beta \mathrm{ST}$ was transformed to a high- $\beta$ ST with $\beta$ of $40 \%$ with an absolute min-B profile [91]. This proof of principle promotes the application of this scheme to a larger ST, MAST.

\section{Plasma Scenario - Real Time Control of MHD, Heating and Current Drive -}

In the topics of the plasma scenario in the present category, highlighted issues are real time control of MHD modes [92-95], high power heating in metallic walls [96-98], and steady-state operation scenario [99-106].

Intelligent real time control has been reported from several experiments. In particular, TCV 
has demonstrated that ECRH triggers individual ELMs independently of preceding ones. For a given trigger sequence, the resulting ELM sequences were highly reproducible (see Fig.17). Localization of ECRH power was a key for high efficiency control with lower power. Failsafe NTM prevention also has been demonstrated by sawtooth pacing by a combination of pre-emptive low-power ECRH on island and backup power for NTM stabilization [92]. DIII-D has reported that real-time control of EC power and mirror steering provided complete stabilization of $m / n=2 / 1$ NTM. Here it should be noted that MSE detection is used for the first time, which has the potential to save required leading time and power compared with other diagnostics [93] A real-time ECRH/ECCD system to control NTMs as well as sawtooth pacing with pulsed ECH has been also reported by FTU [94.95].

Safe application of heating power and high performance scenario development for a metallic wall is a critical issue in the plasma scenario. Achievements of high heating power have been reported from JET [96] and ASDEX-Upgrade [97]. In JET, following the NBI system upgade, power levels of $25.7 \mathrm{MW}$, and pulse length of $15 \mathrm{~s}$ (using a single injection box) were obtained with the ILW. Although all private and surrounding limiters of ICH have been changed to beryllium, 4MW could be coupled in H-mode. Although good heating performance was observed, higher bulk radiation in the ICH plasma than NBI heated plasmas was observed due to higher tungsten and nickel levels. The source of higher tungsten concentration observed with ICRH has not yet been identified [96]. ASDEX-Upgrade has demonstrated high performance discharges with $P / R=14 \mathrm{MW} / \mathrm{m}$. Nitrogen puff to control divertor radiation has enabled heating power of $23 \mathrm{MW}$ with a total radiation of $20 \mathrm{MW}$ including divertor radiation of 9MW. Eventually, the divertor heat load was reduced to less than 5 $\mathrm{MW} / \mathrm{m}^{2}$ at the $\mathrm{H}$ factor of around 1 and $\beta_{\mathrm{N}}=2.8$. ICRH of $4 \mathrm{MW}$ is compatible with the tungsten wall [97]. In LHD, metallic impurities have been suppressed to low levels, i.e., $n_{\mathrm{Fe}} / n_{e}<10-5$, even though the first wall is made of stainless steel. Impurity screening in the stochastic layer surrounding nested flux surfaces worked to suppress the penetration of metallic impurities and this screening effect was more effective for heavier impurities due to their outer ionization front [98].

Steady-state tokamak operation requires broad current and pressure distributions to enable high $\beta_{\mathrm{N}}$. Current distributed off axis is favorable for steady-state, MHD stability and confinement. DIII-D used off- axis NBI to develop and test the scenario for high $\beta_{\mathrm{N}}$ steady state operation. An elaborate NBI facility allowing vertical steering has demonstrated development of a quasi-stationary scenario [99]. Off-axis NBI current drive (NBCD) has produced broad current and pressure profiles with sustained $q_{\min }>2$ for higher $\beta_{\mathrm{N}}(\sim 4)$ stability limit [100] (see Fig.18). It has also demonstrated a quasi-stationary scenario with performance that projects to $\mathrm{Q}=5$ in ITER with $q_{\min } \sim 1.5$ and a current drive fraction due to NBI of $70 \%$ 
for twice the resistive diffusion time. Off-axis NBCD in MAST has indicated that the fast ion distribution is close to the classical picture [106]

Novel approaches of ICRF have also been reported from LHD [103] and Alcator C-Mod $[107,108]$. LHD has reported that the use of dipole phasing gave better heating performance than monopole phasing as predicted by the theory [103]. Alcator C-mode has reported observation of ICRF-induced radial electric field in SOL, which can play role in increase of impurity source and penetration [108], and the successful result from field aligned ICRF antenna to reduce impurity contamination [107]. These observations have suggested the importance of the control of RF field to suppress impurity contamination and to enhance heating efficiency. In this regard, NSTX has reported interesting evidence that RF edge losses suggest fast-wave propagation in SOL and its modeling which was meant to predict and minimize such edge losses in the ICRF heating regime [109].

\section{Waves and Energetic Particles}

A variety of contributions have shown the progress in this topic with regard to fast ion relaxation [106,110], energetic particle driven mode [111-123], electron Bernstein waves [88,124,125], lower hybrid waves [126-129] and fast waves [130].

The experiment of off axis NBI in MAST showed that confinement of fast ions was close to classical and that off-axis NBI broadened fast ion pressure profile, eventually mitigating the growth of fishbone modes and the redistribution of fast ions [106]. In the MST RFP, the analysis of the d-d neutron decay rate due to a tangential NBI with $25 \mathrm{keV}$ has suggested that fast ion confinement is close to classical even in the standard RFP with a stochastic magnetic field 110]. Also several bursty instabilities driven by energetic ions have been observed for the first time in an RFP [110].

The dynamics of energetic particle (EP) driven modes in wall-stabilized high beta plasmas has been investigated by joint experiments of JT-60U and DIII-D. ELM triggering by EP driven modes has been identified for the first time. ELM triggering by EP driven modes occurred with strong distortion $(n \neq 1)$ amplitude, and EP transport to the edge by EP driven modes affected edge ELM stability [111] (see Fig.19). Energetic-ion-driven MHD Instabilities in stellarator/heliotron plasmas with low magnetic shear have been studied in joint experiments of Heliotron-J (low rotational transform) and TJ-II (high rotational transform) with different degree of rotational transforms. The scan of rotational transform has indicated that Global Alfvén Eigen modes (GAEs) are mainly destabilized in low shear helical plasma and Helicity AEs (HAEs) were destabilized in high rotational transform configuration [112]. Heliotron-J 
has also reported that EP driven modes were suppressed when the magnetic shear exceeds the threshold for ECCD in the counter direction[113]. Tilting the NBI in DIII-D also allowed the variation of the AEs drive and tests of stability models [114].

HL-2A has reported multi-mode co-existence of an electron-Beta induced AE (BAE). A new mode is excited in the presence of both strong tearing modes and BAEs with a Geodesic Acoustic Mode (GAM) in Ohmic plasmas. Since the GAM was localized in the core, it was different from one excited by the drift-wave turbulence in the edge plasma [115]. LHD has reported an interesting observation of energy transfer between ions and the GAM. During the burst of the GAM, the energetic ions lost energy, and the ions in the lower energy range gained the energy (see Fig.20). This observation suggested that the GAM can become an efficient energy channel from EPs to bulk ions [116].

Two new approaches using wave heating schemes have been proposed to control the edge plasma. One is an edge magnetic topology change induced by LHCD in EAST. Helical radiation belts led to the splitting of divertor strike points with effects similar to those with an RMP [129]. Another was ICRF in NSTX. Optimized higher harmonic fast waves can drive edge harmonic oscillations with larger displacements [130]. It is expected that the effect of both approaches on the control of ELMs will be assessed in the near future.

\section{Concluding Remarks}

Compared with the achievements reported at the last FEC2010, the understanding of experimental results has progressed remarkably, in particular, in RMP and ILW, which are the highlight of this FEC2012. It should be noted that PWI used to be out of the scope of this series of conference, but now it has become a super critical topic. Together with the recognition of remarkable progress, it was confirmed again that we still need tenacious efforts to get a more accurate and comprehensive knowledge to extrapolate to ITER and DEMO. Typical remaining issues are the significance of "resonance" in ELM suppression/mitigation by RMP, effect of metal walls on the performance of plasma confinement, and documentation of disruptions.

The meaning of "ITER relevant" should be assessed more seriously than before. Identification of correlation is not enough and only identification of the bridging mechanism between the actuator and the consequence, in other words, identification of causality could provide the most reliable extrapolation to ITER/DEMO. The fusion community has to prepare resolutions to these issues in time to lead ITER to success. It should be emphasized that no single experiment can resolve these issues. It is quite important to rally all kindred experiments to 
solve the problem.

\section{Acknowledgements}

It is a great pleasure to thank all contributors for their outstanding achievements, in particular, Dr.W.Baonian, Dr.S.Coda, Dr.X.Duan, Dr.M.Greenwald, Dr.D.Hill, Dr.J.-G.Kwak, Dr.P.Marcin, Dr.H.Meyer, Dr.F.Romanelli, Dr.S.Sabbagh, Dr.J.Sarff, and Prof.U.Stroth who provided representative results from leading experimental devices. Fruitful communications with Prof.K.Itoh, Prof.K.Ida, Dr.Y.Kamada, Prof.O.Kaneko and Dr.N.Asakura are gratefully acknowledged. The author has tried to extract the most important topics and highlighted new results to fulfill the Terms of Reference to summary speakers. Therefore, the author expresses his sincere acknowledgements to all contributors to this 24th IAEA FEC2012 even though their papers have not been taken up explicitly here.

\section{References}

References presented below as papers at this conference are available online at http://www-naweb.iaea.org/napc/physics/FEC/FEC2012/html/index.htm

[1] Jaquinot, J. 2011 Nucl. Fusion 51094001

[2] Loarte, A. et al Progress on the application of ELM control schemes to ITER, ITR/1-2 FEC 2012, San Diego

[3] Greenfield, C.M. et al, 2011 Nucl. Fusion 51094009

[4] Liang, Y. et al 2011 Nucl. Fusion 51073001

[5] Lloyd, B. et al 2011 Nucl. Fusion 51094013

[6] Canik, J.M. et al 2010 in Fusion Energy 2010 (Proc.23rd Int. Conf. Daejon, 2010) (IAEA:Vienna) CD-ROM file EXC/8-1 and http://www.naweb.iaea.org/napc/physics/FEC/FEC2010/html/index.htm

[7] Suttrop, W. et al Mitigation of edge localised modes with small mon-axisymmetric magnetic perturbations in ASDEX Upgrade, EX/3-4 FEC 2012, San Diego

[8] Jeon, Y.M. et al ELM control in application of non-axisymmetric magnetic perturbations in KSTAR, EX/3-3 FEC 2012, San Diego

[9] Toi, K. et al Mitigation of large amplitude edge-localized-modes by resonant magnetic perturbations on the large helical device, EX/P4-10 FEC 2012, San Diego

[10] Wade, M.R. et al Advances in the physics understanding of ELM suppression using resonant magnetic perturbations in DIII-D, EX/3-1 FEC 2012, San Diego

[11] Kirk, A. et al Understanding ELM mitigation by resonant magnetic perturbations on MAST, Submitted to Nuclear Fusion (December 2012)

[12] Park, H.J. et al Visualization of ELM dynamics and its response from external 
perturbations via 2D electron cyclotron emission imaging in KSTAR, EX/11-3 FEC 2012, San Diego

[13] Liang, Y. et al Mitigation of type-I ELMs with $\mathrm{n}=2$ Fields on JET, Submitted to Nuclear Fusion (January 2013)

[14] Orlov, D.M. et al 2012 Fusion Eng. Design 871536

[15] Fishpol, G. et al MAST contributions to the exhaust challenge, including testing of super-X, EX/P5-17 FEC 2012, San Diego

[16] Kočan, M. et al Far-reaching impact of intermittent transport across the scrape-off layer: latest results from ASDEX upgrade, Submitted to Nuclear Fusion (January 2013)

[17] Suzuki, Y. et al 3D plasma response to magnetic field structure in the Large Helical Device, Submitted to Nuclear Fusion (January 2013)

[18] Sakakibara, S. et al Response of MHD stability to resonant magnetic perturbation in the Large Helical Device, Submitted to Nuclear Fusion (January 2013)

[19] Suzuki, Y. et al Nucl. 2006 Nucl. Fusion 46 L19.

[20] Buttery, R.J. et al Addressing new challenges for error field correction, EX/P4-31 FEC 2012, San Diego

[21] Frassinetti, L. et al Experimental studies of the plasma response to applied nonaxisymmetric external magnetic perturbations in EXTRAP T2R, EX/P4-21 FEC 2012, San Diego

[22] Garcia-Munoz, M. et al Fast-ion redistribution and loss due to edge perturbations in the ASDEX Upgrade, DIII-D and KSTAR Tokamaks, EX/P6-03 FEC 2012, San Diego

[23] Levesque, J. et al High resolution detection and 3D magnetic control of the helical boundary of a wall-stabilized tokamak plasma, Submitted to Nuclear Fusion (January 2013)

[24] Masamune, S. et al Direct observation of soft-X ray filament structure and high current operation in low-aspect-ratio RFP, EX/P4-24 FEC 2012, San Diego

[25] Chapman, B. et al Direct diagnosis and parametric dependence of 3D helical equilibrium in the MST RFP, EX/P6-01 FEC 2012, San Diego

[26] Maingi, R. et al The nearly continuous improvement of discharge characteristics and edge stability with increasing lithium coatings in NSTX, EX/11-2 FEC 2012, San Diego

[27] Soukhanovskii, V. et al Snowflake divertor as plasma-material interface for future high power density fusion devices, EX/P5-21 FEC 2012, San Diego

[28] Vijvers, W. A. J. et al Reduction of peak wall power loads in L- and H-mode tokamak plasmas in TCV with the snowflake divertor, EX/P5-22 FEC 2012, San Diego

[29] Allen, S.L. et al Initial snowflake divertor physics studies on DIII-D, PD/1-2 FEC 2012, San Diego

[30] Morisaki, T. et al First results of closed helical divertor experiment in LHD, Submitted to Nuclear Fusion (January 2013) 
[31] Hill, D.N. et al DIII-D overview - Research toward resolving key issues for ITER and steady-state Tokamaks, Submitted to Nuclear Fusion (November 2012)

[32] Wischmeier, $M$. et al Symmetries and asymmetries in the divertor detachment in ASDEX upgrade, EX/P5-34 FEC 2012, San Diego

[33] Giroud, C. et al Nitrogen seeding for heat load control in JET ELMy H-mode plasmas and its compatibility with ILW materials, EX/P5-30 FEC 2012, San Diego

[34] Petrie, T. W. et al Effect of changes separatrix magnetic geometry on divertor behavior in DIII-D, EX/P5-12 FEC 2012, San Diego

[35] Kobayashi, M. et al Control of $3 \mathrm{D}$ edge radiation structure with resonant magnetic perturbation fields applied to stochastic layer and stabilization of radiative divertor plasma in LHD, EX/4-4 FEC 2012, San Diego

[36] Ahn, J.-W. et al Toroidal asymmetry of divertor heat deposition during the ELM and 3-D field application in NSTX, EX/P5-33 FEC 2012, San Diego

[37] Merten, P. et al Power handling of the tungsten divertor in JET, EX/P5-24 FEC 2012, San Diego

[38] Wang, L. et al Characterization of particle and power loads on divertor targets for type-I, type-III, and mossy ELMy H-modes in EAST superconducting tokamak, Submitted to Nuclear Fusion (December 2012)

[39] Makowski, M.A. et al Scaling of the divertor heat flux width in the DIII-D tokamak, EX/P5-16 FEC 2012, San Diego

[40] Vianello, N. et al 3D effects on RFX-mod helical boundary region, EX/P8-02 FEC 2012, San Diego

[41] Makowski, M.A. et al 2012 Phys. Plasmas 19056122

[42] Romaneli, F. et al Overview of the JET Results with the ITER-like Wall, Submitted to Nuclear Fusion (November 2012)

[43] Brezinsek, S. et al Fuel retention studies with the ITER-like wall in JET, Submitted to Nuclear Fusion (November 2012)

[44] Roth, J. et al 2008 Plasma Phys. Control. Fusion 50103001

[45] Tsitrone, E. et al In Vessel fuel inventory build-up in tokamaks: Lessons learnt from Tore Supra, EX/P5-07 FEC 2012, San Diego

[46] de Varies, P. et al Comparison of plasma breakdown with a carbon and ITER-like wall, Submitted to Nuclear Fusion (November 2012)

[47] Coenen, J.W. et al Longterm evolution of the impurity composition and transient impurity events with the ITER-like wall at JET, Submitted to Nuclear Fusion (January 2013)

[48] Douai, D. et al Overview of the international research on ion cyclotron wall conditioning, EX/P5-09 FEC 2012, San Diego

[49] Mirnov, S. et al Recent achievements of the T-11M lithium program, EX/P5-25 FEC 


\section{2, San Diego}

[50] Gray, $\mathrm{T}$, et al The effects of increasing lithium deposition on the power exhaust channel in NSTX, Submitted to Nuclear Fusion (January 2013)

[51] Jaworski, M. et al Liquid lithium divertor characteristics and plasma-material interactions in NSTX high-performance plasmas, Submitted to Nuclear Fusion (January 2013)

[52] Tabares, F. et al Studies of plasma-lithium interactions in TJ-II, Submitted to Nuclear Fusion (January 2013)

[53] Puiatti, M.E. et al Wall conditioning and density control in the reversed field pinch RFX-mod, Submitted to Nuclear Fusion (January 2013)

[54] Zhang, L. et al Effect of lithium coating on edge particle recycling in EAST H-mode discharge, EX/P5-02 FEC 2012, San Diego

[55] Wagner, F. et al 1991 in Plasma Physics and Controlled Nuclear Fusion Research 1990 (Proc.13th Int. Conf. Washington DC, 1990) Vol.1, p.277, IAEA, Vienna (1991)

[56] Itoh, K. and Itoh, S.-I. 1995 Plasma Phys. Control. Fusion 37491

[57] Wright, G. et al Assessment of tungsten nano-tendril growth in the Alcator C-Mod divertor, EX/P5-03 FEC 2012, San Diego

[58] Kajita, S. et al Impact of arcing on carbon and tungsten: from the observations in JT-60U, LHD, and NAGDIS-II, Submitted to Nuclear Fusion (January 2013)

[59] Koga, K. et al Control of Dust Flux in LHD and in a Divertor Simulator, EX/P5-32 FEC 2012, San Diego

[60] van Rooij, G. et al Characterization of tungsten sputtering in the JET divertor, EX/P5-05 FEC 2012, San Diego

[61] Takamura, S. et al Cooling characteristics and mitigation of He-defected tungsten with nanostructure formation, EX/P5-06 FEC 2012, San Diego

[62] Rudakov, D. L. et al Measurements of net versus gross erosion of molybdenum divertor surface in DIII-D, EX/P5-11 FEC 2012, San Diego

[63] Philipps, V. et al Development of laser based techniques for in-situ characterization of the first wall in ITER and future fusion devices, EX/P5-14 FEC 2012, San Diego

[64] Greenwald, M. et al Overview of Experimental Results and Code Validation Activities at Alcator C-Mod, Submitted to Nuclear Fusion (November 2012)

[65] Rubel, M. et al Efficiency of fuel removal: Overview of techniques tested on plasma-facing components from the TEXTOR Tokamak, EX/P5-10 FEC 2012, San Diego

[66] Hollmann E.M. et al Control and dissipation of runaway electron beams created during rapid shutdown experiments in DIII-D, EX/9-2 FEC 2012, San Diego

[67] Saint-Laurent, F., et al Overview of runaway electrons control and mitigation experiments on Tore Supra and lessons learned in view of ITER, EX/P8-06 FEC 2012, 
San Diego

[68] Plyushin, V. V. et al Latest progress in studies of runaway electrons in JET, EX/P8-05 FEC 2012, San Diego

[69] Pautasso, G. et al Towards the density required for runaway electron suppression in ITER, EX/P8-12 FEC 2012, San Diego

[70] Zhang, Y. et al Study of runaway electron generation in synergetic ECRH \& SMBI plasma and during major disruptions in the HL-2A tokamak, EX/P8-15 FEC 2012, San Diego

[71] Kwak, J.-G et al Overview of KSTAR Results Submitted to Nuclear Fusion (December 2012)

[72] Lehnen, M. et al Impact and mitigation of disruptions with the ITER-like wall in JET, EX/9-1 FEC 2012, San Diego

[73] Berkery, J. et al Global mode control and stabilization for disruption avoidance in high-beta NSTX plasmas, EX/P8-07 FEC 2012, San Diego

[74] Volpe, F. A. et al Stabilization of disruptive locked modes by magnetic perturbations and electron cyclotron current drive at DIII-D, EX/P8-08 FEC 2012, San Diego

[75] Granetz, R. et al Disruption mitigation experiments with two gas jets on Alcator C-Mod, EX/P8-09 FEC 2012, San Diego

[76] Gerhardt, S. et al Disruptions in the high-beta spherical torus NSTX, Submitted to Nuclear Fusion (September 2012)

[77] Murari, A. et al Latest developments in data analysis tools for disruption prediction and for the exploration of multimachine operational spaces, EX/P8-04 FEC 2012, San Diego

[78] Savrukhin, P. et al Fast-scale magnetic perturbations and onset of the energy quench during disruption instability in the T-10 tokamak, EX/P4-34 FEC 2012, San Diego

[79] Park, Y.-S. et al Investigation of plasma rotation alteration and MHD stability in the expanded H-mode operation of KSTAR, Submitted to Nuclear Fusion (December 2012)

[80] Lin, Y. et al Characterization of neo-classical tearing modes in high-performance I-Mode Plasmas with ICRF mode conversion flow drive on Alcator C-Mod, Submitted to Nuclear Fusion (January 2013)

[81] Xiao, C. et al Modification of toroidal flow in the STOR-M tokamak, EX/P4-26 FEC 2012, San Diego

[82] Lazzaro, E. et al Triggerless Onset and effect of "natural" rotation on neoclassical tearing modes in the TCV Tokamak, EX/P4-32 FEC 2012, San Diego

[83] Seol, J.-C. et al Effects of RF-heating induced MHD activities on the toroidal rotation in tokamaks, EX/P8-11 FEC 2012, San Diego

[84] Kim, J.-H. et al The Effect of toroidal plasma rotation on sawtooth activity in KSTAR, Submitted to Nuclear Fusion (January 2013)

[85] Gusev, V. et al From Globus-M results toward compact spherical tokamak with 
enhanced parameters, Globus-M2, Submitted to Nuclear Fusion (January 2013)

[86] Takase, Y. et al Non-inductive Plasma initiation and plasma current ramp-up on the TST-2 spherical tokamak, Submitted to Nuclear Fusion (January 2013)

[87] Zushi, H. et al Non-inductive current start-up and plasma equilibrium with an inboard poloidal field null by means of electron cyclotron waves in QUEST, EX/P2-14 FEC 2012, San Diego

[88] Uchida, M. et al Noninductive formation of spherical tokamak at 7 times the plasma cutoff density by electron Bernstein wave heating and Current Drive on LATE, EX/P6-18 FEC 2012, San Diego

[89] Raman, R. et al Non-inductive plasma start-up in NSTX using transient CHI, Submitted to Nuclear Fusion (January 2013)

[90] Redd, A. et al Local helicity injection startup and edge stability studies in the pegasus toroidal experiment, EX/P4-36 FEC 2012, San Diego

[91] Ono, Y. et al High power heating of magnetic reconnection for high-beta ST formation in TS-3 and UTST ST merging experiments, EX/8-2 FEC 2012, San Diego

[92] Duval, B. et al Real time ELM, NTM and sawtooth control on TCV, EX/1-2 FEC 2012, San Diego

[93] Koleman, E. et al State-of-the-art neoclassical tearing mode control in DIII-D using realtime steerable electron cyclotron current drive launchers, PD- FEC 2012, San Diego

[94] Sozzi, C. et al First operations of the real-time ECRH/ECCD system for control of magnetohydrodynamics instabilities in the FTU tokamak, EX/P6-04 FEC 2012, San Diego

[95] Nowak, S. et al Control of sawteeth periods by pulsed ECH and ECCD in FTU tokamak, Submitted to Nuclear Fusion (January 2013)

[96] Mayoral, M.L. et al On the challenge of plasma heating with the JET metallic wall, EX/4-3 FEC 2012, San Diego

[97] Bobkov, V. et al ICRF operation with improved antennas in a full W-wall ASDEX upgrade, status and developments, Submitted to Nuclear Fusion (January 2013)

[98] Morita, S. et al Low concentration of iron as first wall material in LHD plasmas with edge ergodic layer, EX/P5-18 FEC 2012, San Diego

[99] Park, J. M. et al Validation of off-axis neutral beam current drive physics in the DIII-D tokamak EX/P2-13 FEC 2012, San Diego

[100] Holcomb, C.T. et al Fully noninductive scenario development in DIII-D using new off-axis neutralbeam injection, EX/1-5 FEC 2012, San Diego

[101] Gong, X. et al Integrated operation scenarios for long pulse discharges in EAST, EX/P2-06 FEC 2012, San Diego

[102] Jackson, G.L. et al Long-pulse stability ILimits of ITER baseline scenario plasmas in DIII-D, EX/P2-08 FEC 2012, San Diego 
[103] Mutoh, T. et al Steady state operation using improved ICH and ECH for high performance plasma in LHD, Submitted to Nuclear Fusion (January 2013)

[104] Shiraiwa, S. et al Progress toward steady-state regimes in Alcator C-Mod, Submitted to Nuclear Fusion (January 2013)

[105] Guo, H. et al Approaches towards steady-state advanced divertor operations on EAST by active control of plasma-wall interactions, Submitted to Nuclear Fusion (January 2013)

[106] Turnyanskiy, M. et al Measurement and optimisation of the fast ion distribution on MAST, Submitted to Nuclear Fusion (January 2013)

[107] Wukitch, S. et al Evaluation of optimized ICRF and LHRF antennas in Alcator C-Mod, FTP/1-1 FEC 2012, San Diego

[108] Terry, J. et al The Fine-scale Structure of the radial electric field in the scrape-off-layer during ICRF heating in Alcator C-Mod, EX/P5-39 FEC 2012, San Diego

[109] Perkins, R.J. et al Fast wave power flow along SOL field lines in NSTX, Submitted to Nuclear Fusion (January 2013)

[110] Anderson, J.K. et al Classical confinement of impurity ions and NBI-born fast ions in the reversed field pinch, EX/P3-16 FEC 2012, San Diego

[111] Matsunaga, G. et al Dynamics of energetic particle driven modes and MHD modes in wall-stabilized high beta plasmas on JT-60U and DIII-D, EX/5-1 FEC 2012, San Diego

[112] Yamamoto, S. et al Studies of energetic-ion-driven MHD instabilities in helical plasmas with low magnetic shear, EX/5-2 FEC 2012, San Diego

[113] Nagasaki, K. et al Stabilization of energetic-ion-driven MHD ode by ECCD in Heliotron J, EX/P8-10 FEC 2012, San Diego

[114] Heidbrink, W.W. et al Fast ion physics enabled by off-Axis neutral beam injection, EX/P6-22 FEC 2012, San Diego

[115] Chen, W. et al Observation of GAM induced by energetic electrons and NL interactions among GAM, BAEs and tearing modes on the HL-2A Tokamak, Submitted to Nuclear Fusion (December 2012)

[116] Ido, T. et al Observation of a new energy channel from energetic particles to bulk ions through geodesic acoustic mode, PD/P8-16 FEC 2012, San Diego

[117]Croker, N., Internal amplitude, structure and identification of CAEs and GAEs in NSTX, Submitted to Nuclear Fusion (December 2012)

[118] Fredrickson, E. et al Fast-ion energy loss during TAE avalanches in the National Spherical Torus Experiment, EX/P6-05 FEC 2012, San Diego

[119] Guimaraes Filho, Z. et al Electron fishbones in LHCD plasmas on FTU and Tore Supra, EX/P6-07 FEC 2012, San Diego

[120] Hole, M.J. et al Analysis of Alfvén wave activity in KSTAR plasmas, EX/P6-09 FEC 2012, San Diego 
[121] Yu, J. et al Frequency jump phenomena of e-fishbone during high power ECRH on HL-2A tokamak, Submitted to Nuclear Fusion (November 2012)

[122] Ding, X. et al Coexistence of Alfvénic modes induced by energetic electrons with ECRH on HL-2A, EX/P6-15 FEC 2012, San Diego

[123] Blackwell, B. et al MHD activity in the Alfvén range of frequencies in the H-1NF heliac, EX/P6-23 FEC 2012, San Diego

[124] Yoshimura, Y. et al Electron Bernstein wave heating and electron cyclotron current drive by use of upgraded ECH system in LHD, Submitted to Nuclear Fusion (January 2013)

[125] Idei, H. et al Electron Bernstein wave heating and current drive effects in QUEST, EX/P6-17 FEC 2012, San Diego

[126] Ding, B. et al Investigations of LHW-plasma coupling and current drive in H-mode experiments in EAST, Submitted to Nuclear Fusion (January 2013)

[127] Hillariet, J. et al Recent progress on lower hybrid current drive and implications for ITER, Submitted to Nuclear Fusion (October 2012)

[128] Cesario, R. et al Spectral broadening and lower hybrid current drive in high density tokamak plasmas, Submitted to Nuclear Fusion (December 2012)

[129] Liang, Y. et al Edge magnetic topology change induced by LHCD and its profound effects in EAST, PD/P8-09 FEC 2012, San Diego

[130] Park, J.-K. et al Observation of edge harmonic oscillation in NSTX and theoretical study of its active control using HHFW antenna at audio frequencies, Submitted to Nuclear Fusion (January 2013)

Table I Summary of experimental observation of ELM suppression/mitigation by 'R'MP

\begin{tabular}{|c|c|c|c|l|c|}
\hline Device & coils & mode & $\begin{array}{c}\text { type of } \\
\text { perturbation }\end{array}$ & \multicolumn{1}{|c|}{ Experimental findings } & Ref. \\
\hline DIII-D & internal & $\mathrm{n}=3$ & Resonant & $\begin{array}{l}\text { Spatial structure of perturbed } \\
\text { 'effective } T_{e}^{\prime} \text { ' was measured. Its } \\
\text { parity does not indicate island } \\
\text { formation. }\end{array}$ & {$[10]$} \\
\hline MAST & internal & $\mathrm{n}=3,4,6$ & Resonant & $\begin{array}{l}\text { Observed lobe structure suggests } \\
\text { screening effect of plasma }\end{array}$ & {$[11]$} \\
\hline $\begin{array}{c}\text { ASDEX } \\
\text {-Upgrade }\end{array}$ & internal & $\mathrm{n}=1,2,4$ & $\begin{array}{l}\text { Resonant and } \\
\text { Non-resonant }\end{array}$ & $\begin{array}{l}\text { Both 'resonant' and 'non-responat' } \\
\text { perturbations are effective in } \\
\text { suppressing ELMs. }\end{array}$ & {$[7]$} \\
\hline
\end{tabular}




\begin{tabular}{|c|c|c|c|l|c|} 
KSTAR & internal & $\mathrm{n}= \pm 1$ & $\begin{array}{l}\text { Resonant and } \\
\text { Non-resonant }\end{array}$ & $\begin{array}{l}\text { Resonant perturbation suppresses } \\
\text { ELMs while non-resonant } \\
\text { perturbation does not. }\end{array}$ & {$[8]$} \\
\hline LHD & external & $\mathrm{n}=1$ & Resonant & $\begin{array}{l}\text { Resonance exists in the stochastic } \\
\text { layer outside the last closed magnetic } \\
\text { surface, but ELMs are suppressed. }\end{array}$ & {$[9]$} \\
\hline JET & external & $\mathrm{n}=2$ & Non-resonant & $\begin{array}{l}\text { Type-I ELMs transits to small and } \\
\text { high fequency ELMs }\end{array}$ & {$[13]$} \\
\hline
\end{tabular}

\section{Figure Captions}

Fig.1 Discharge waveforms in DIII-D to demonstrate sustained RMP ELM suppression to match the ITER base line scenario [10].

Fig.2 Identification of change of topology due to an RMP in DIII-D. Tangential view of soft X-ray data (left) and inverted poloidal cross-section (right) [10].

Fig.3 Tangential view of lobe structure observed with an RMP in MAST. Top:camera image in the experiment. Bottom: modelled image including screening effect [11].

Fig.4 ECE imaging shows no edge filamentary structure when an ELM is suppressed at 4.3s in KSTAR[12].

Fig.5 Comparison of the radial gradient of the radial electric field and magnetic fields calculated by 3-D MHD equilibrium code HINT2 in LHD [17].

Fig.6 Threshold of the amplitude of RMP (the coil current normalized by the toroidal field) from shield to penetration as a function of magnetic shear in LHD [18].

Fig.7 Effect of flux expansion to snowflake on divertor heat flux and ELMs in DIII-D [29].

Fig.8 Formation of stable radiative divertor by RMP in LHD [35]. (a) Radiation power as function of line-averaged density in LHD. In the case with an RMP, radiation is stabilized against the density. (b) Radiation profile on the poloidal cross-section with an RMP (simulated by EMC3(Ref. Y.Feng et al., Contrib. Plasma Phys.44 (2004)57)-EIRENE(Ref. D.Reiter, et al., Fusion Sci.Technol. 47 (2005) 172).

Fig.9 Comparison of retention rate evaluated by gas balance measurement in different conditions on JET [43].

Fig.10 Evolution of effective CIII concentration in CFC in black and in ILW in red on JET [47]. The index of abscissas; JPN is the pulse number.

Fig.11 Tungsten FUZZ grown in a tokamak (Alcator C-Mod) and a linear device (Pilot-PSI) are identical under similar conditions (temperature, flux, influence, etc.) [57].

Fig.12 High-Z gas injection increases runaway electron dissipation on DIII-D. (a) Massive Gas Injection before the thermal quench and (b) Massive Gas Injection after the 
thermal quench [66].

Fig.13 Parameter regimes at the disruption on the planes of radiated energy vs. plasma energy (left) and force vs. impulse (right) on JET. The cases with CFC, ILW without Massive Gas Injection (MGI) and ILW with MGI are shown in blue, red and green symbols, respectively [72].

Fig.14 Operational trajectories on the plane of the Resonant Field Amplification(RFA) with $n=1$ and $\beta_{\mathrm{N}} / l_{\mathrm{i}}$ on NSTX. Resonant field amplification indicates proximity to marginal stability [73].

Fig. $153 / 2 \& 2 / 1$ tearing modes are characterized with their dependence on plasma rotation on KSTAR [79]. Plasma rotation shear is found to have stabilizing effect on $2 / 1$ mode.

Fig.16 Non-inductive plasma start-up in NSTX using transient Co-axial Helicity Injection (CHI). CHI started discharge in NSTX ramps to $1 \mathrm{MA}$ using $65 \%$ of the inductive flux used by a standard inductive discharge [89].

Fig.17 Demonstration of real-time ELM control on TCV [92].

Fig.18 Off-Axis NBI produces broad current and pressure profiles with sustained $q_{\min }>2$ for higher $\beta_{\mathrm{N}}$ stability limits on DIII-D [100].

Fig.19 ELM triggering by Energetic Particle (EP) driven modes is observed in DIII-D (left) and JT-60U (right) [111]. Associated the EP driven modes are EP driven Wall Mode (EWM) in JT-60U and the off-axis Fishbone Mode (OFM) in DIII-D.

Fig.20 Energy distribution function measured by the Neutral Particle Analyze (NPA) during the GAM bursts in LHD [116]. Variations in the energy distribution function from the spectrum in the range between $60-175 \mathrm{keV}$. The energy of the NBI is $175 \mathrm{keV}$. The burst starts at $4.425 \mathrm{~s}$. 


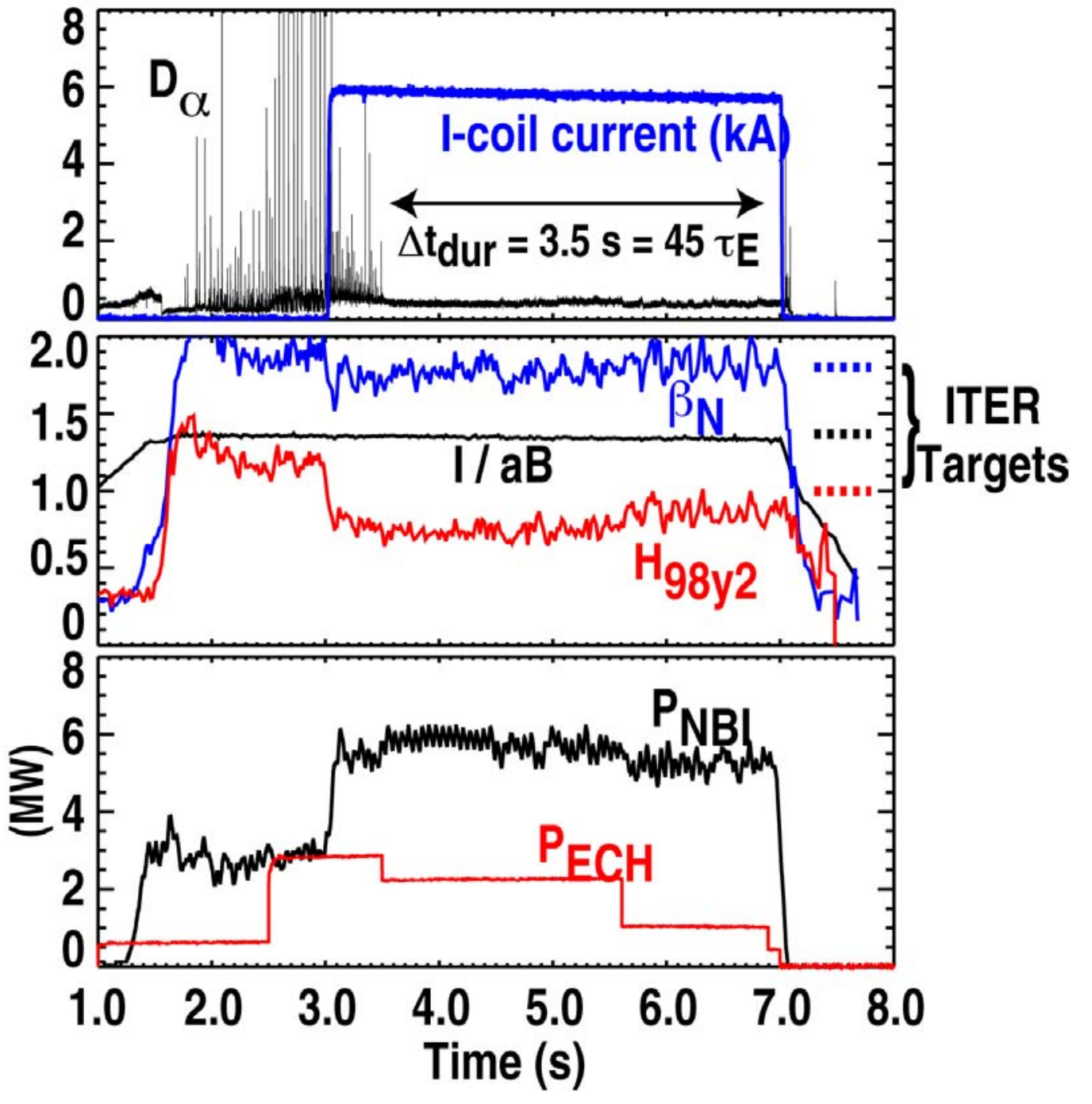

Fig.1
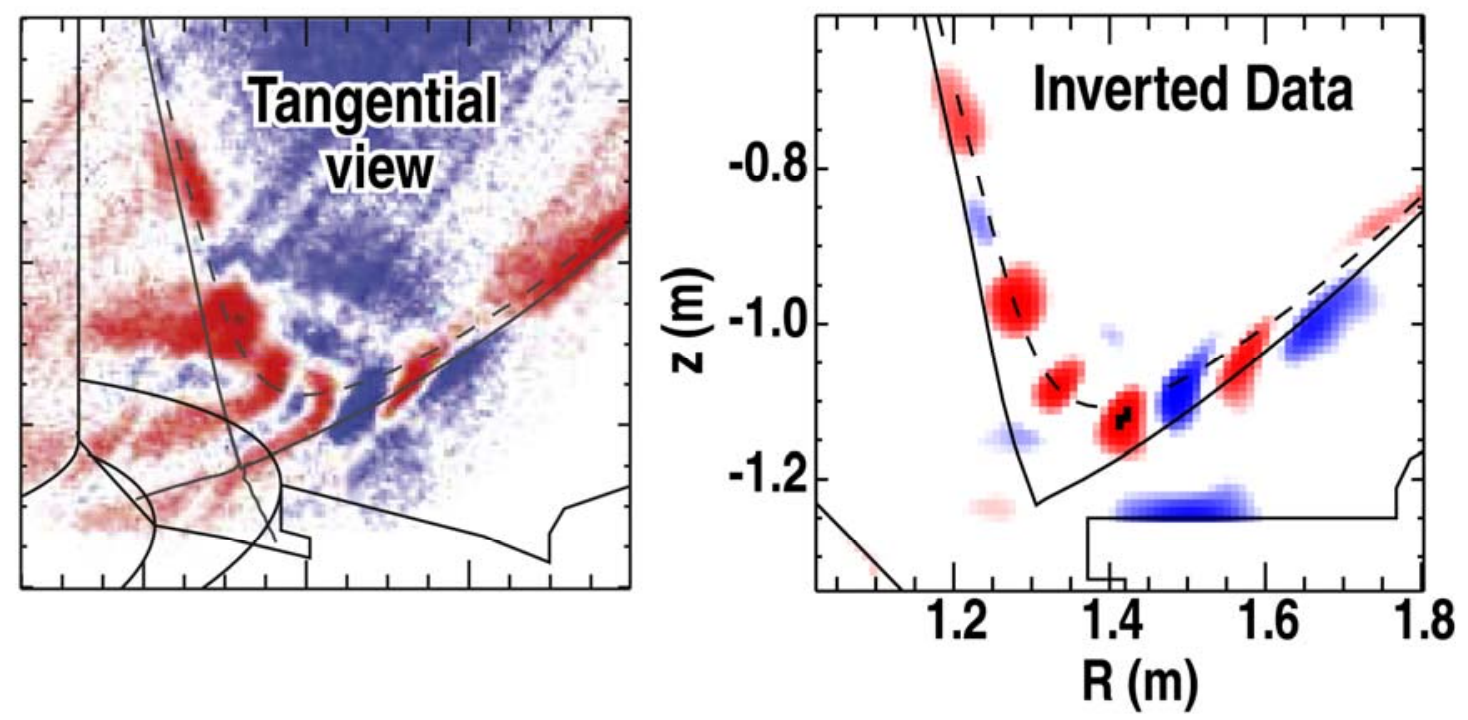
Fig. 2

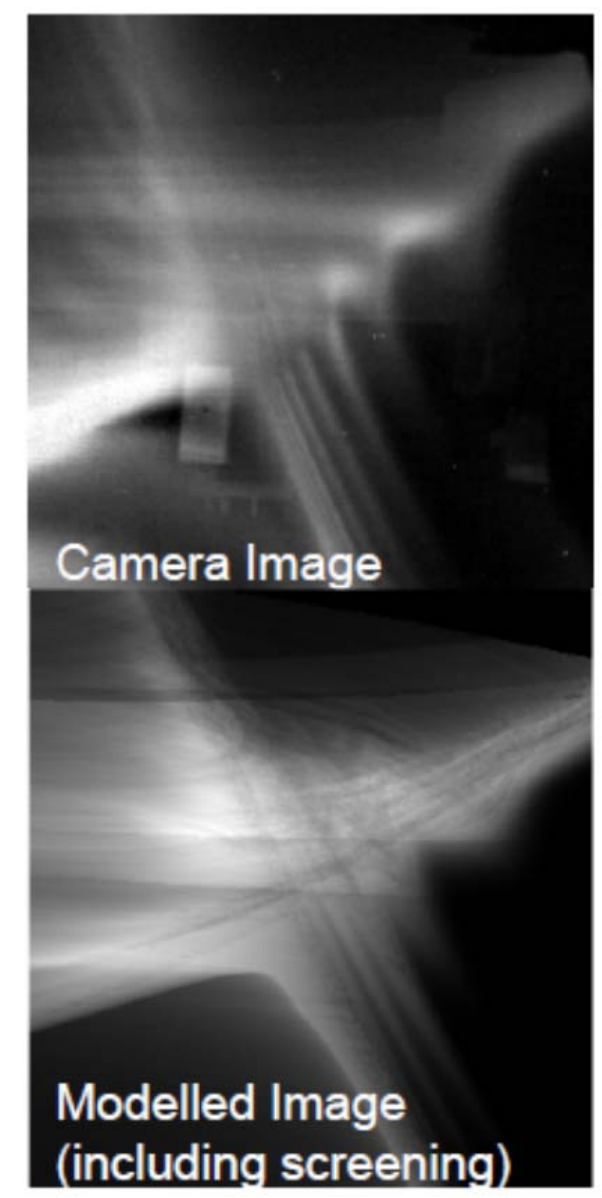

Fig. 3

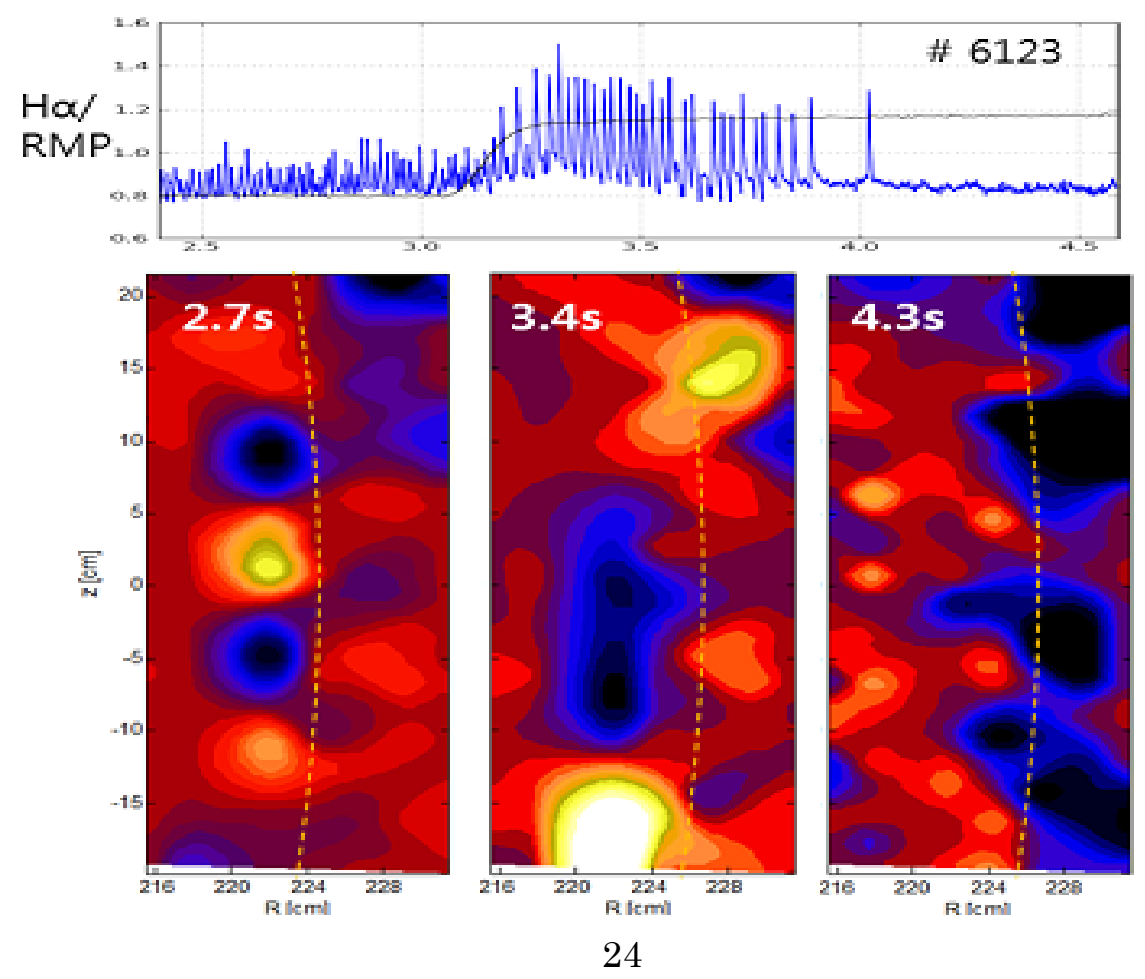


Fig.4

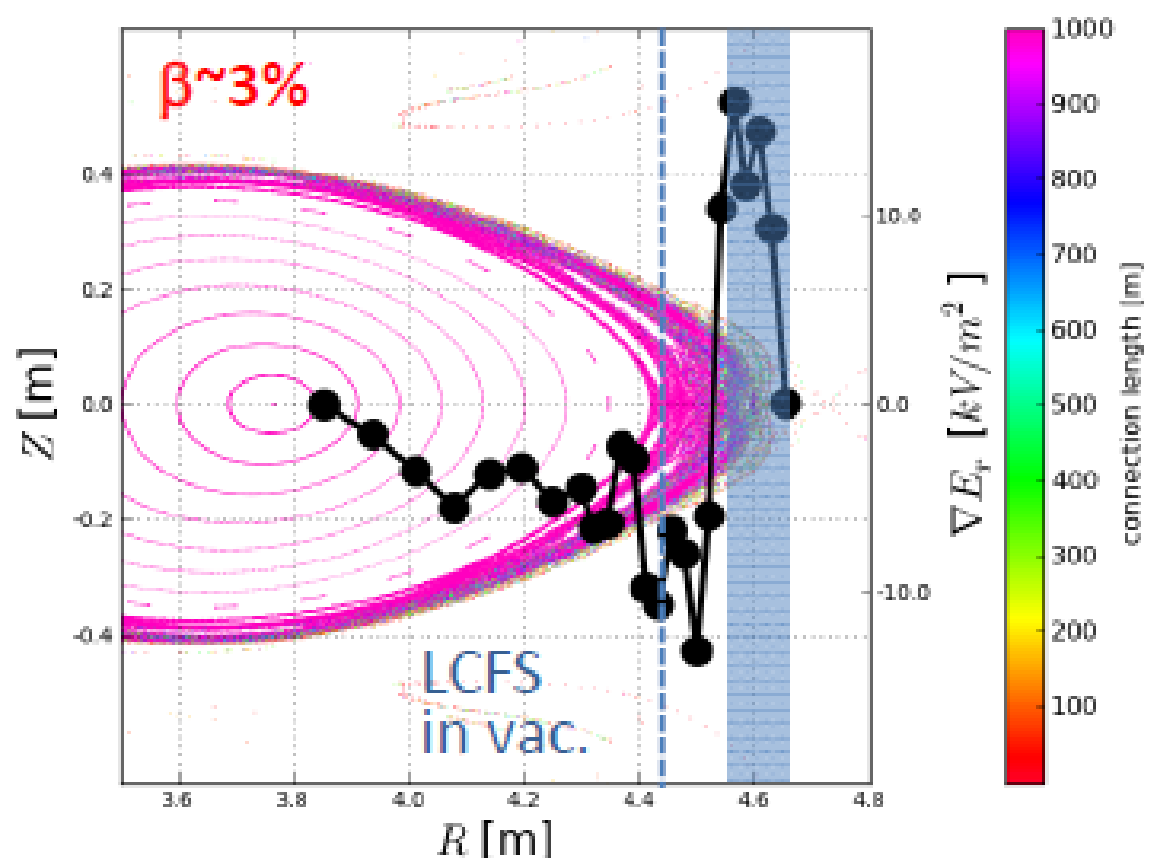

Fig. 5

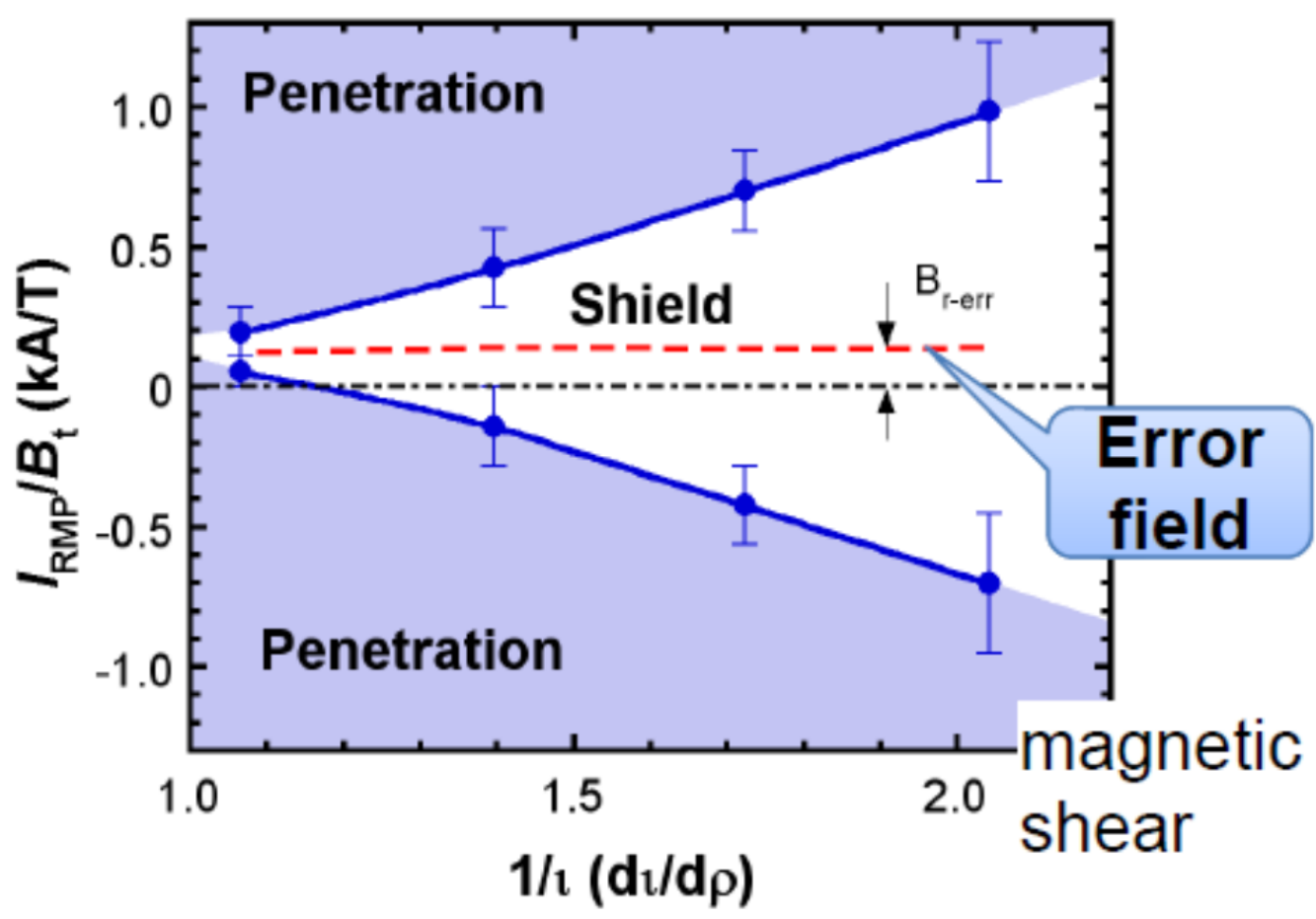

Fig.6 


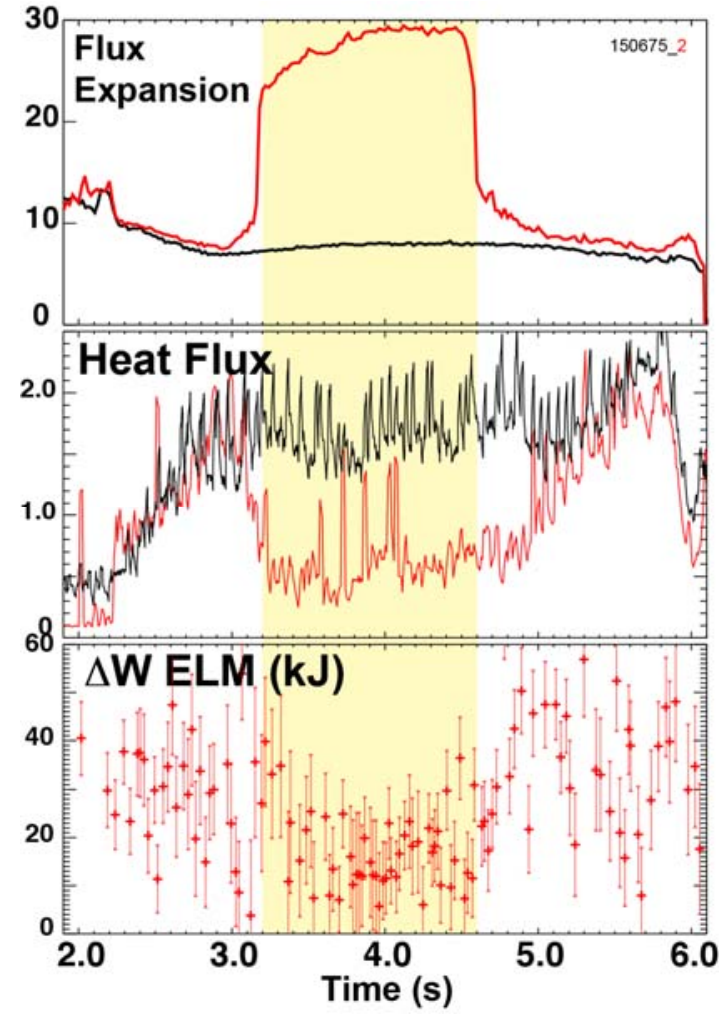

Fig. 7 

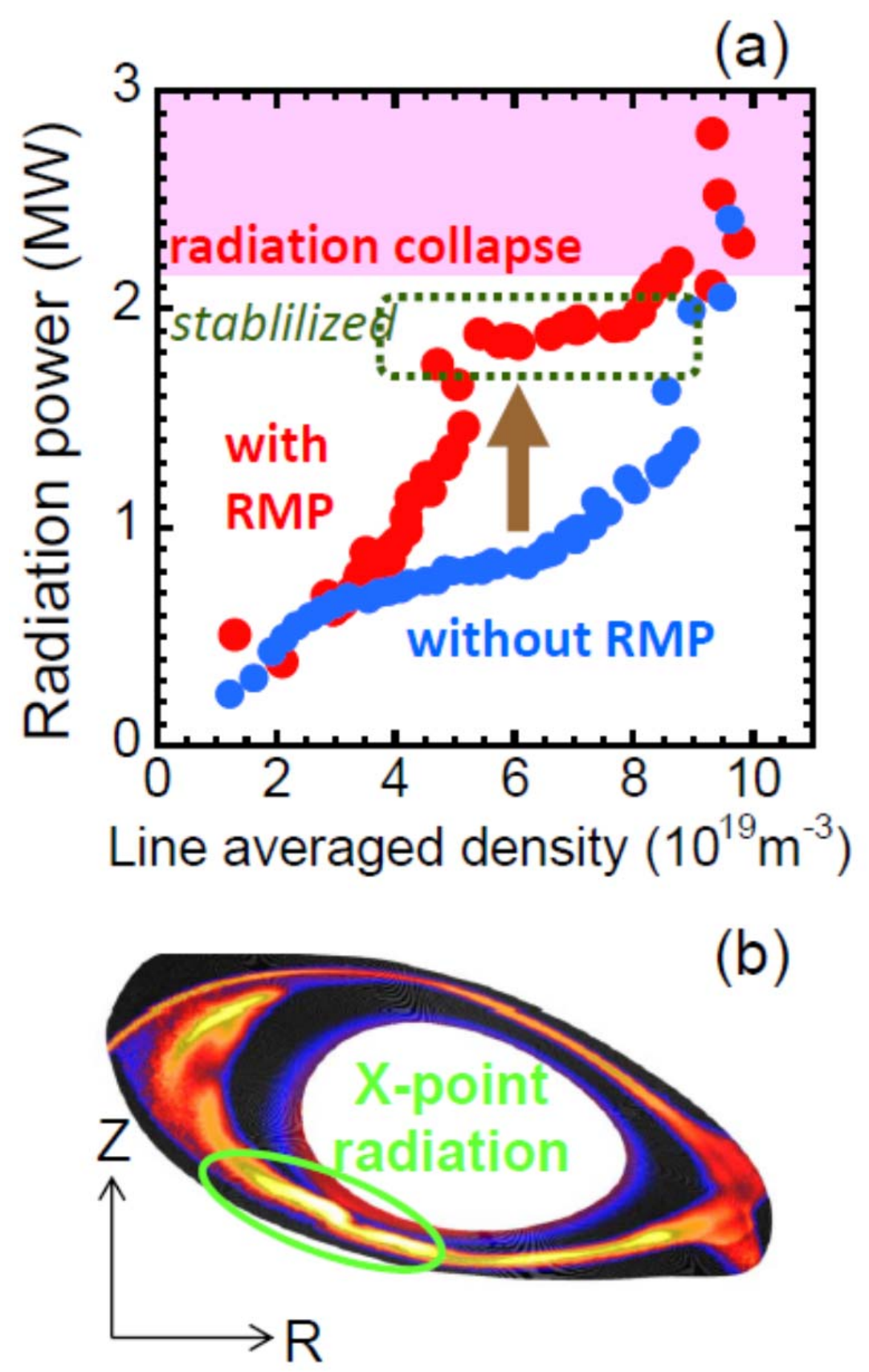

Fig. 8 


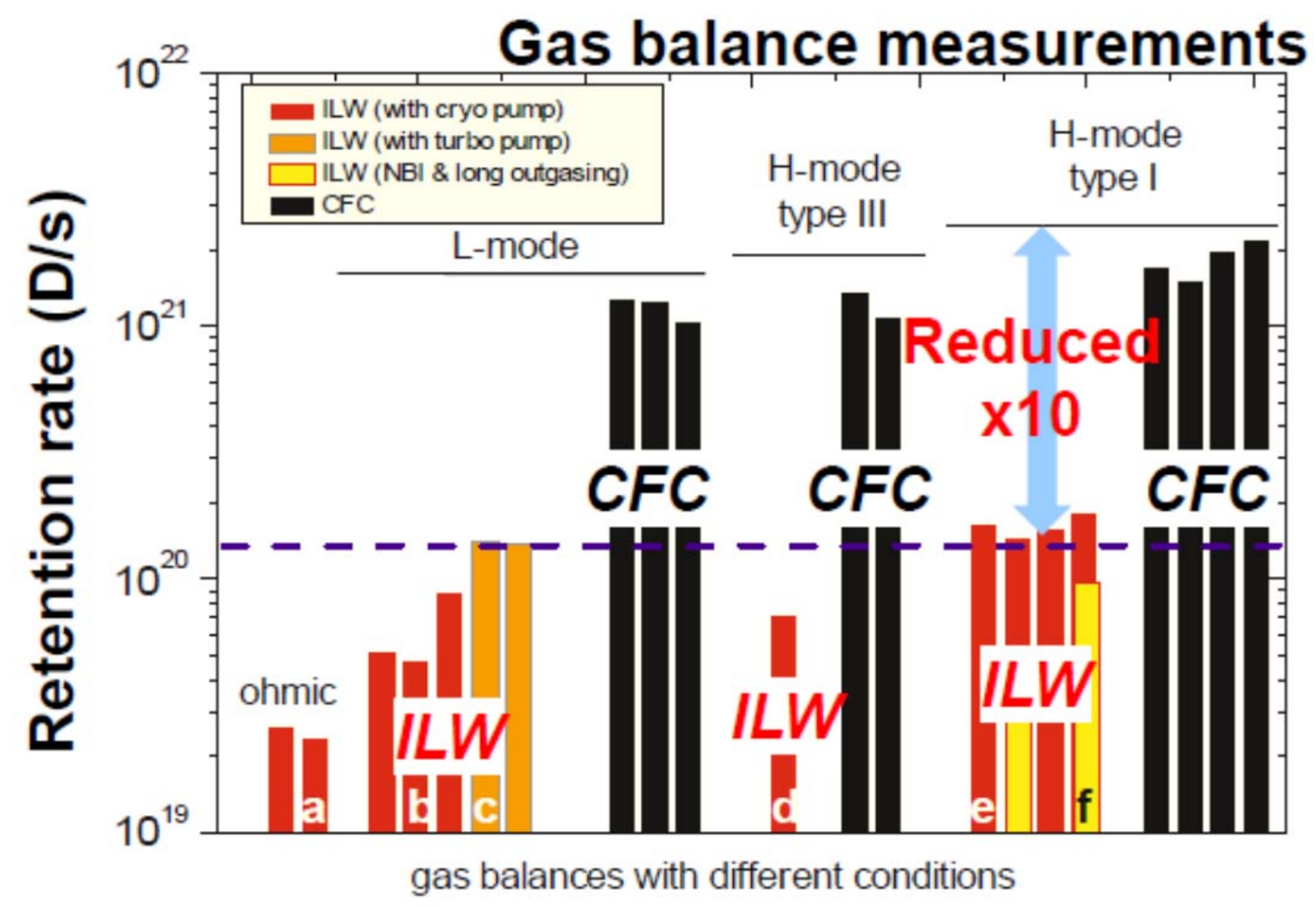

Fig.9

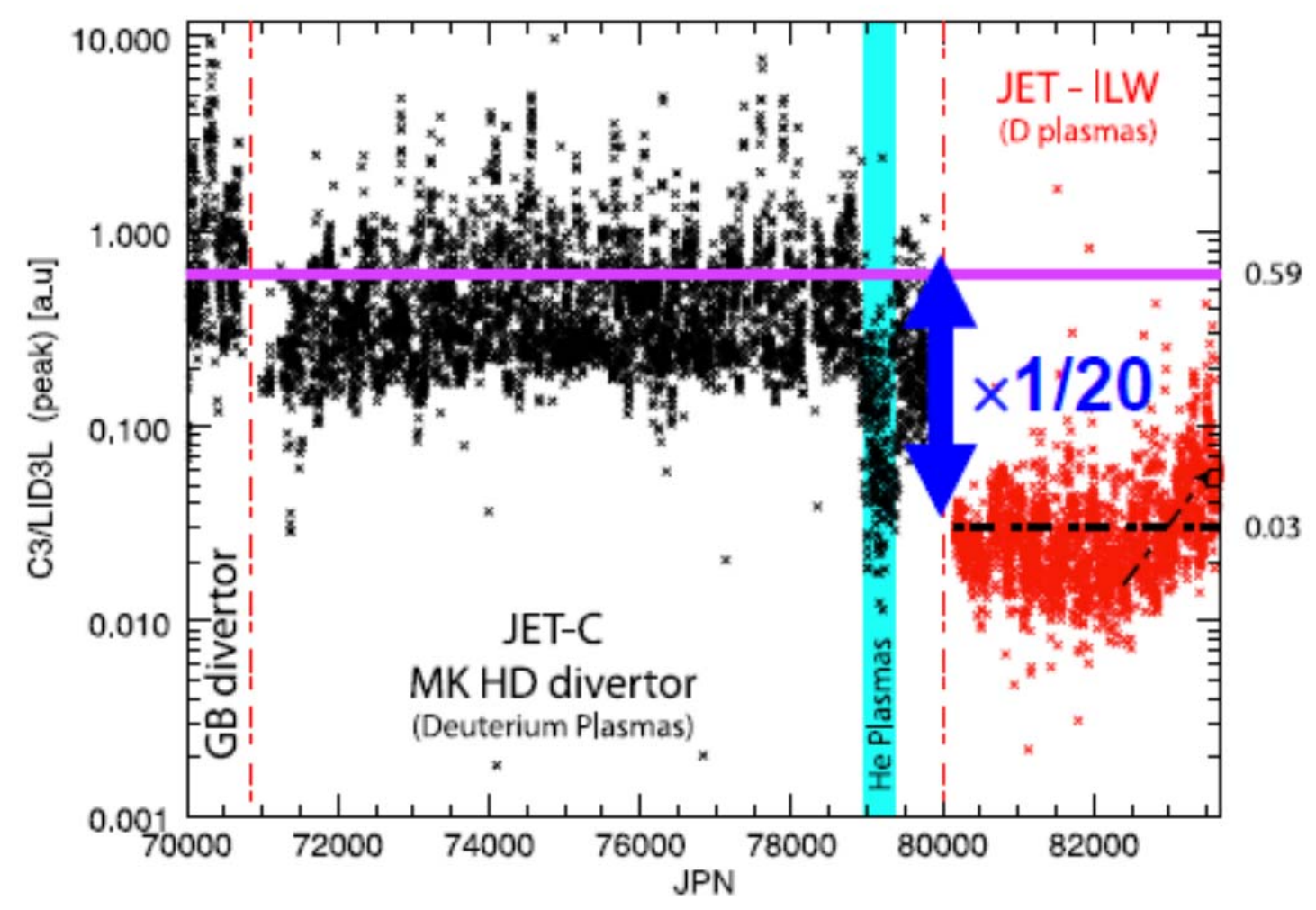

Fig.10 


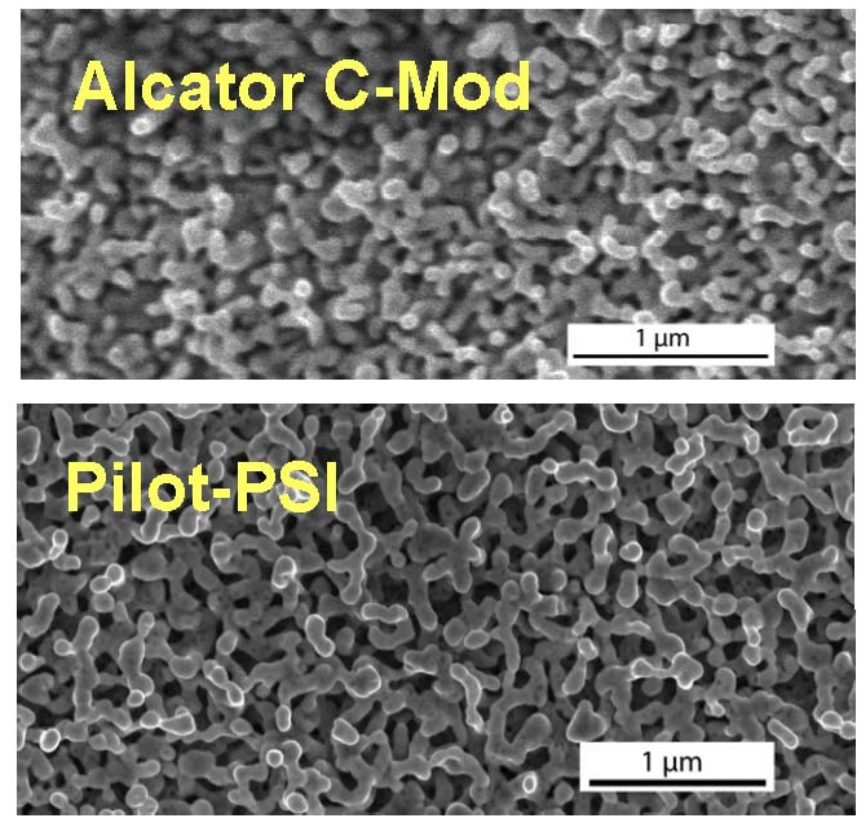

Fig.11

(a) MGI before

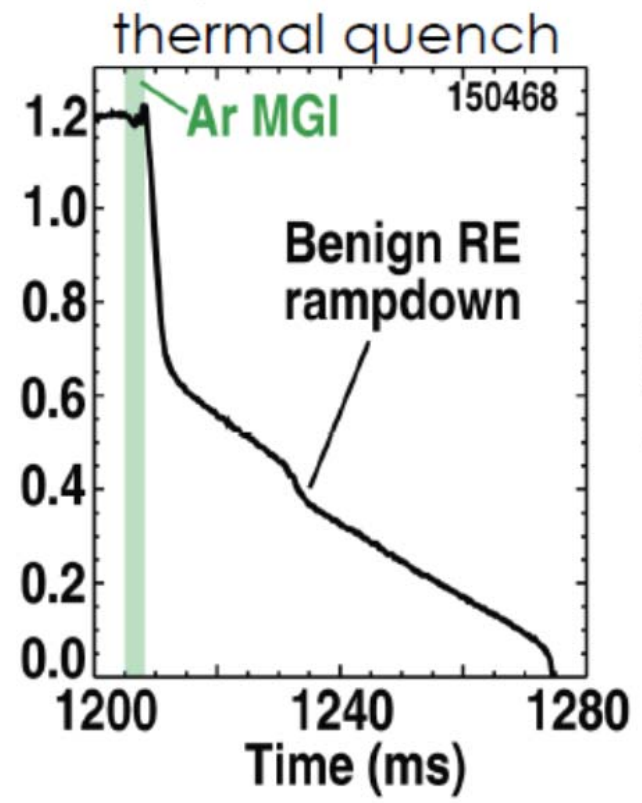

(b) MGl after

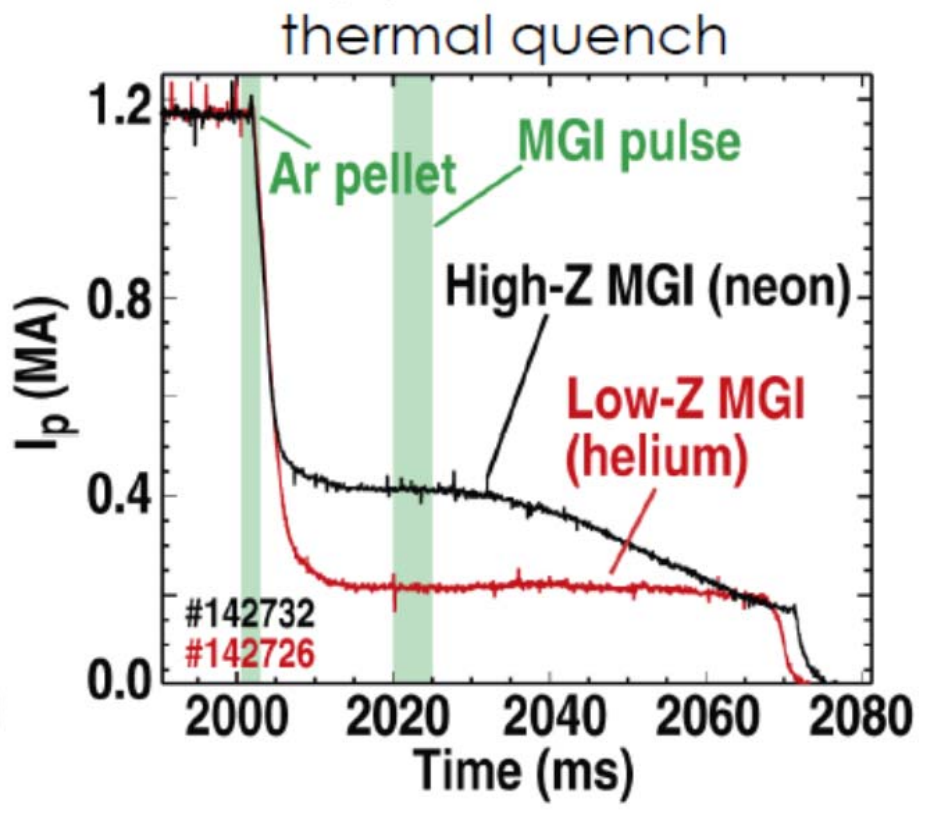

Fig. 12 

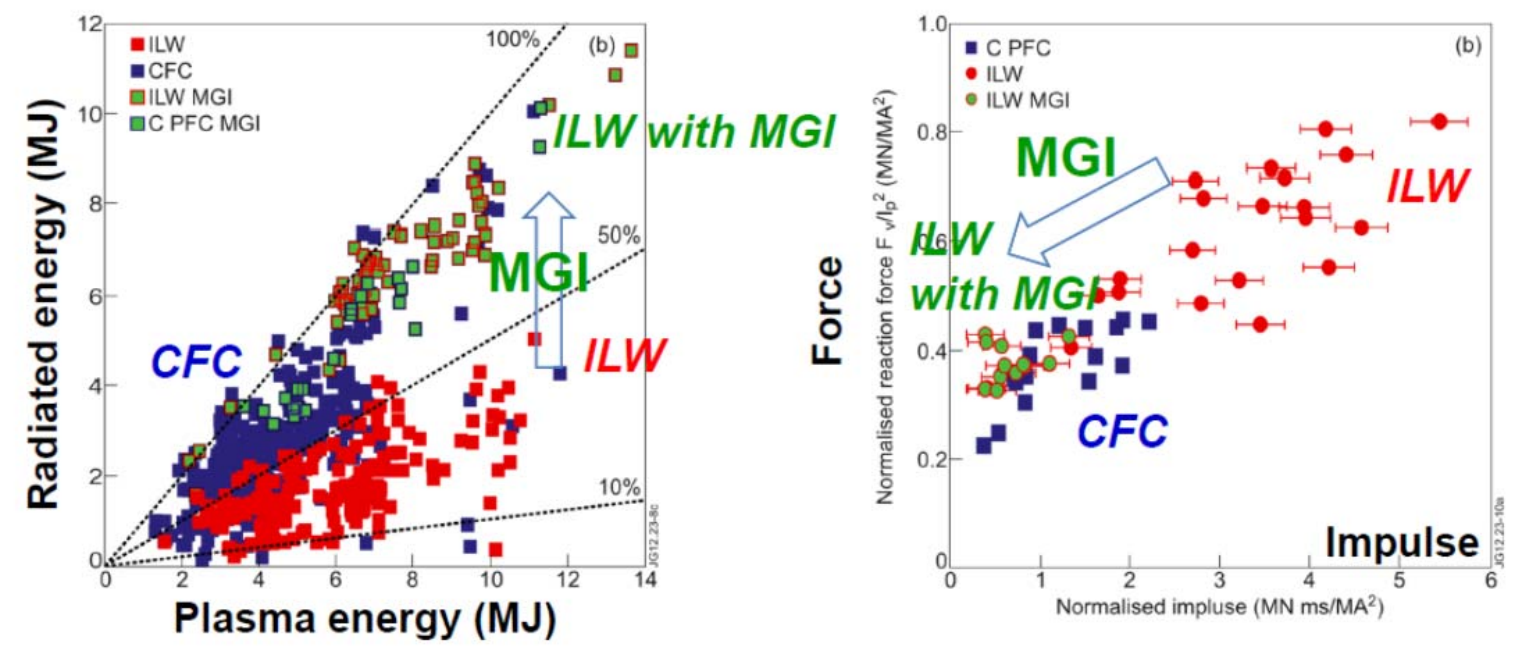

Fig.13

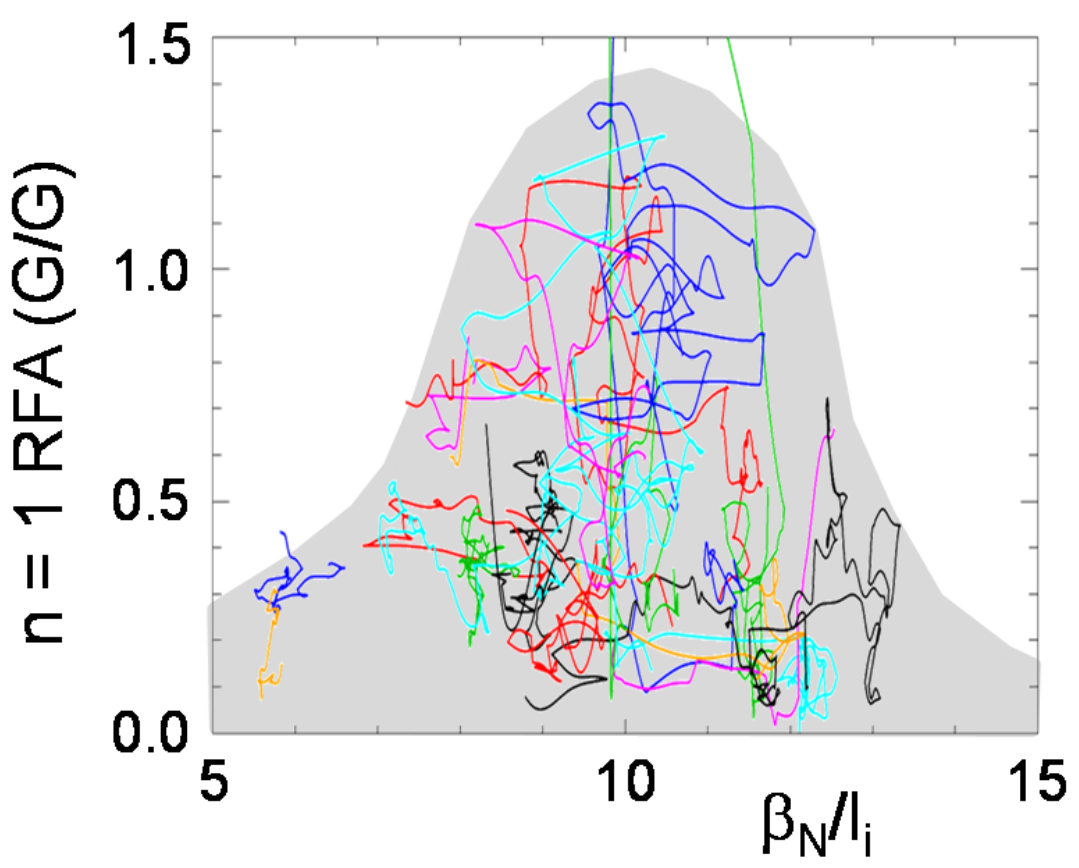

Fig.14 


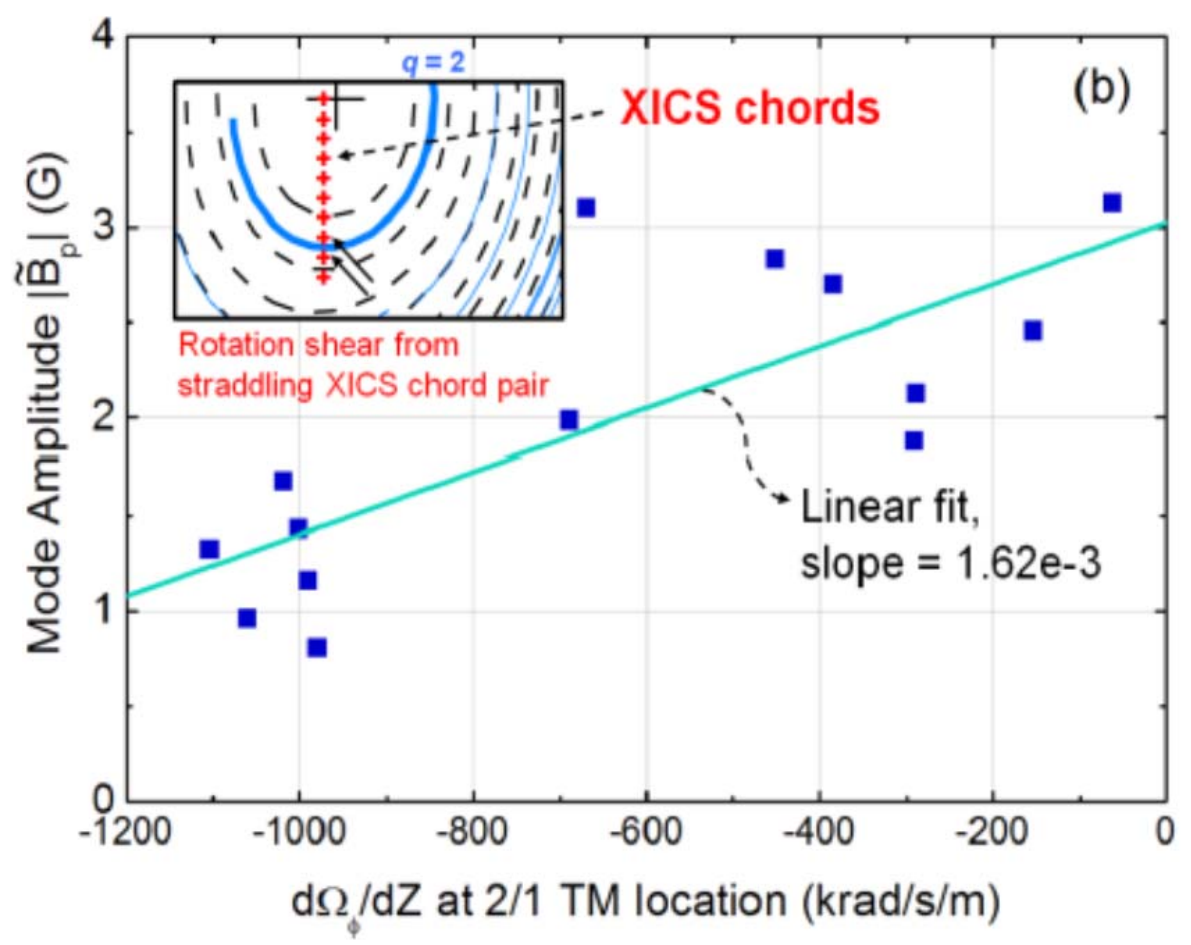

Fig.15

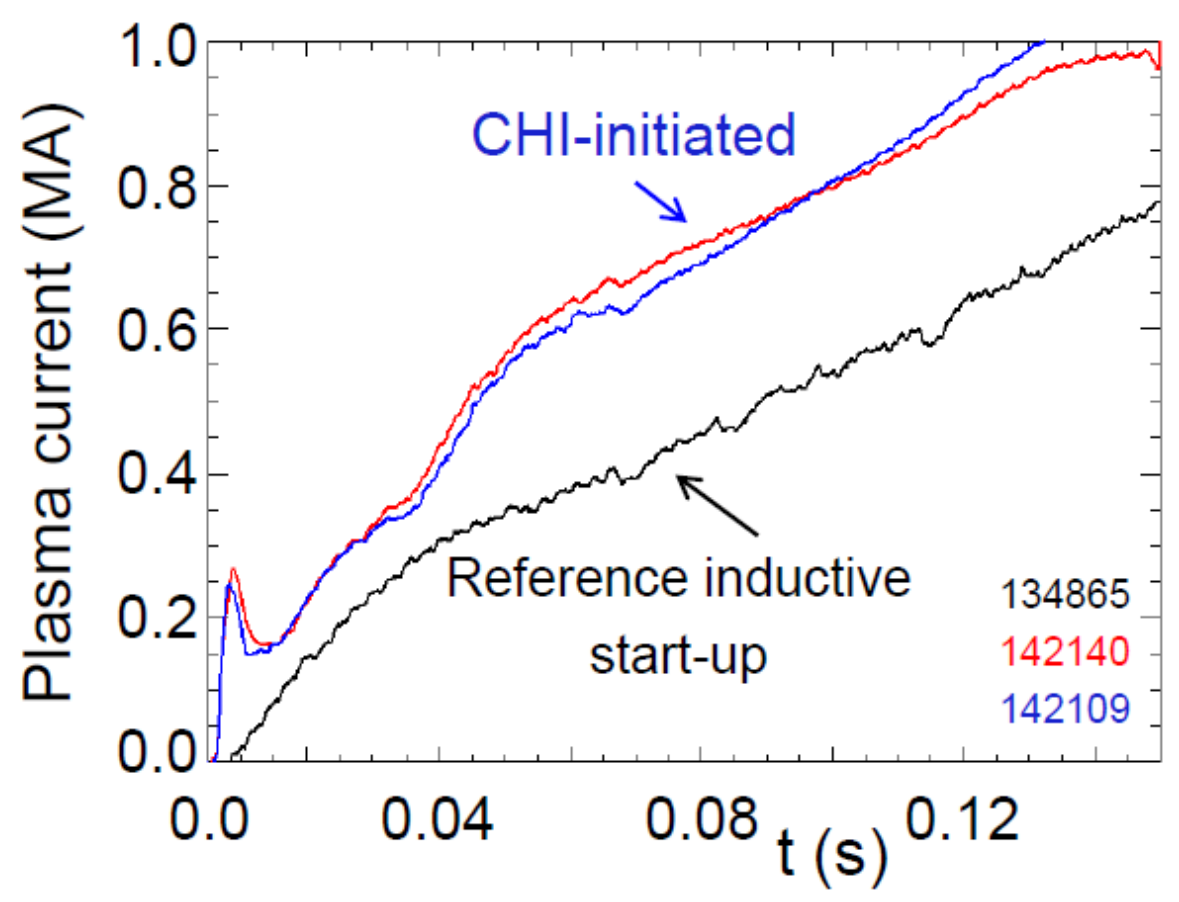

Fig. 16 


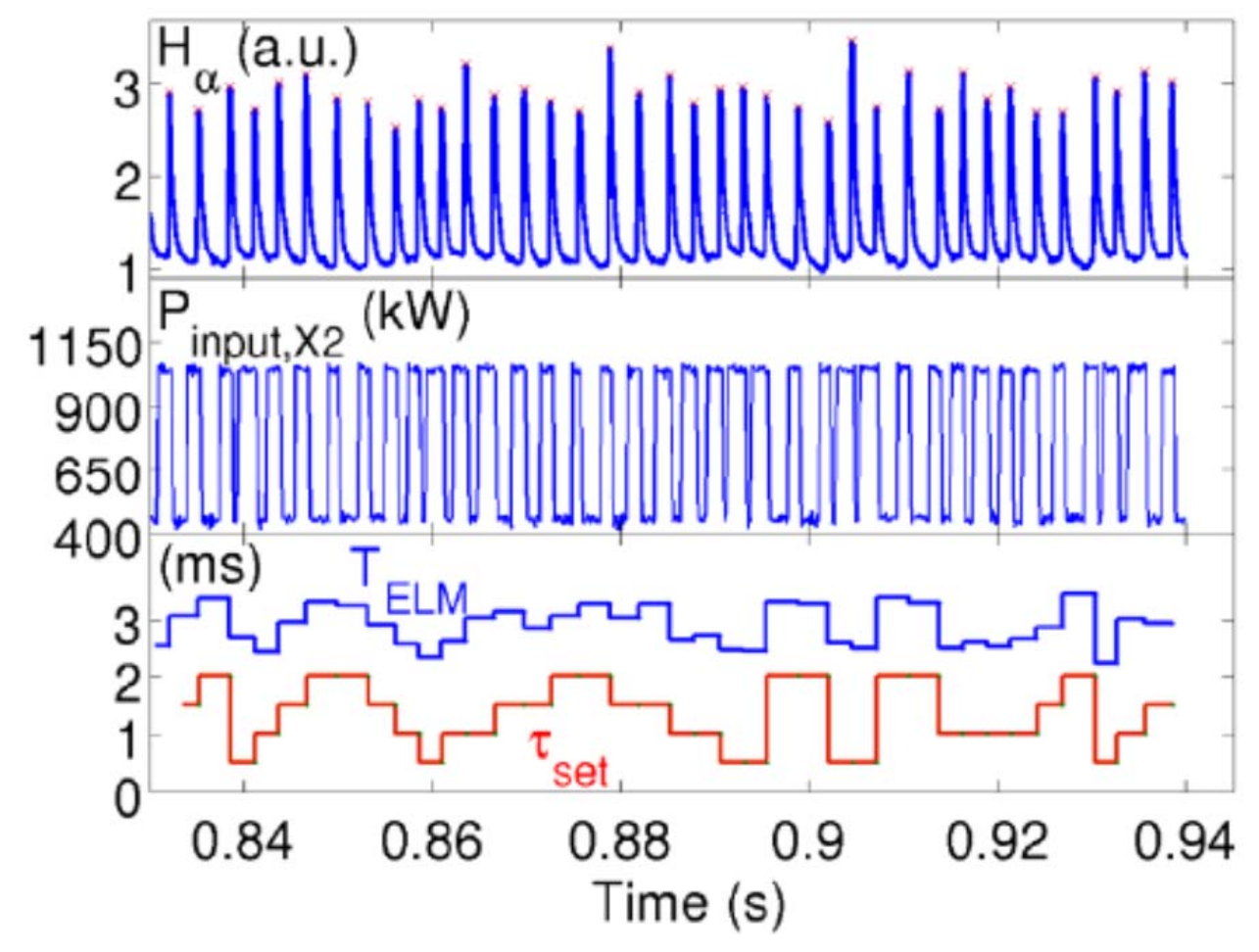

Fig. 17
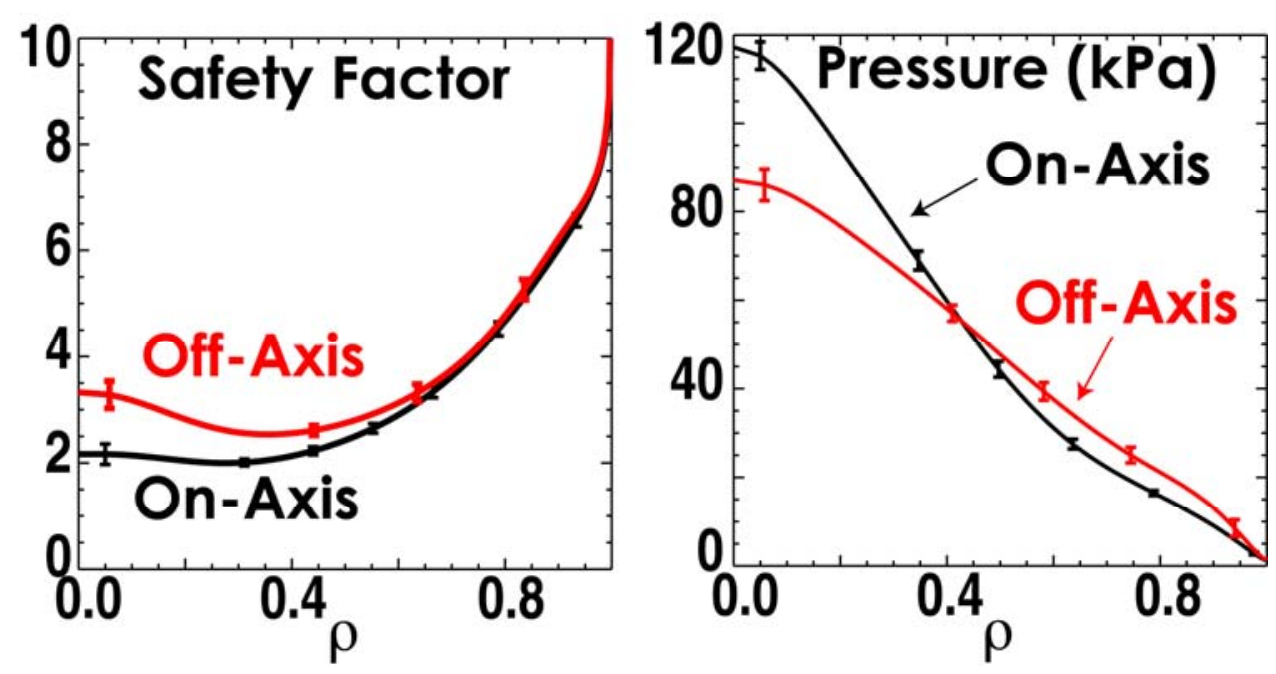

Fig. 18 


\section{DIII-D}
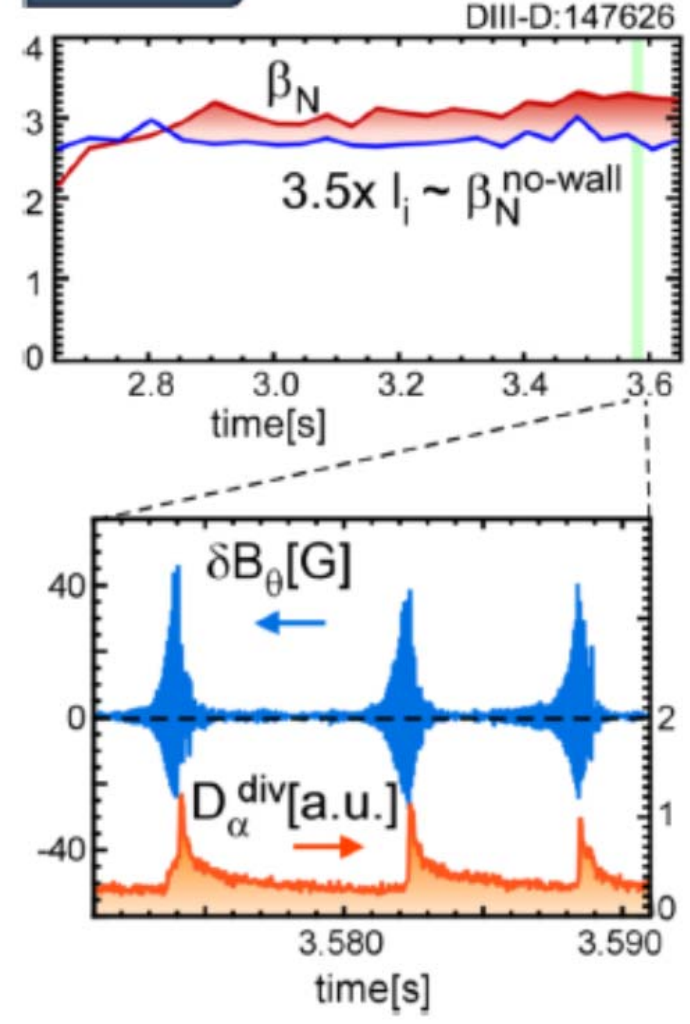

\section{JT-60U}
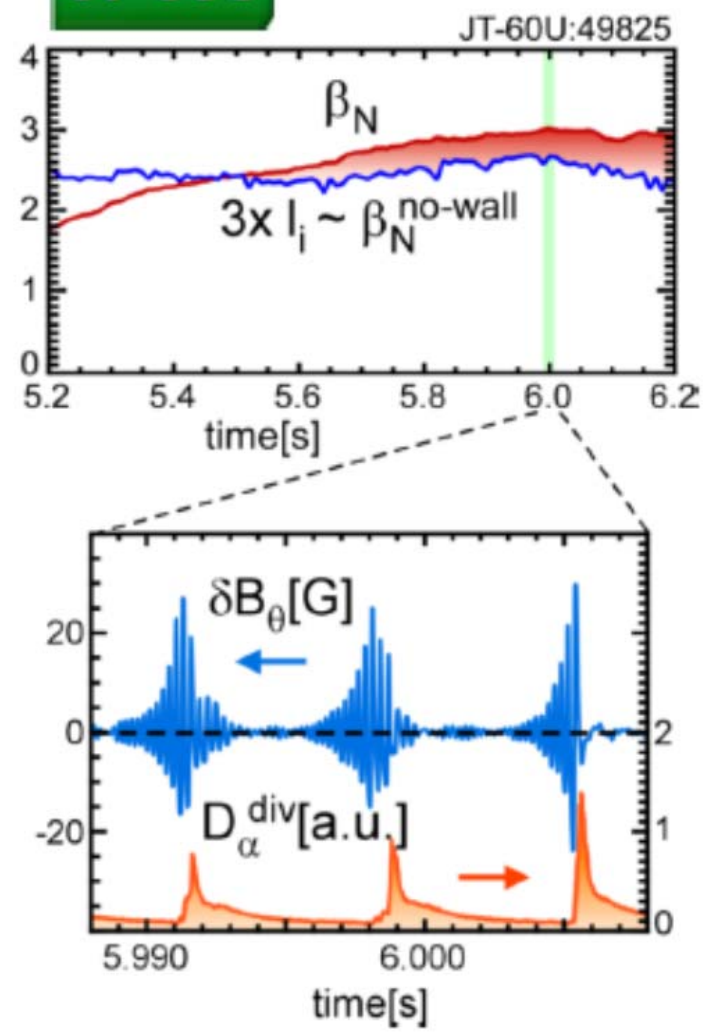

Fig.19

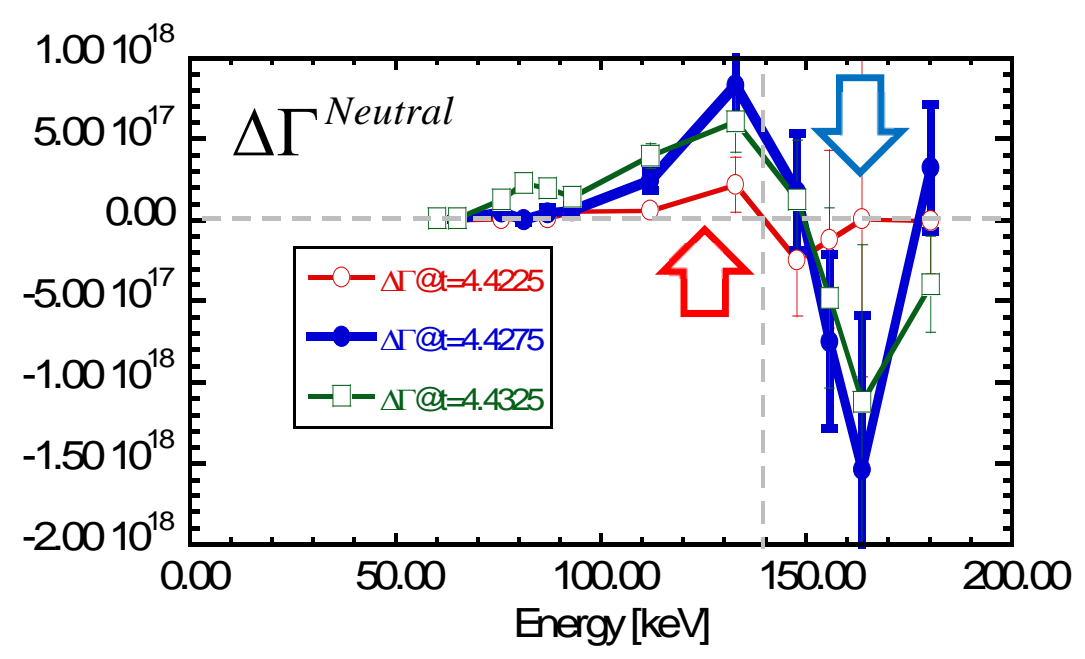

Fig.20 